K. Shimomura

Nagoya Math. J.

Vol. 158 (2000), 133-166

\title{
THE DETERMINATION OF CALORIC MORPHISMS ON EUCLIDEAN DOMAINS
}

\author{
KATSUNORI SHIMOMURA
}

Dedicated to Professor Masayuki Itô in honour of his sixtieth birthday

\begin{abstract}
Let $D$ be a domain in $\mathbb{R}^{m+1}$ and $E$ be a domain in $\mathbb{R}^{n+1}$. A pair of a smooth mapping $f: D \rightarrow E$ and a smooth positive function $\varphi$ on $D$ is called a caloric morphism if $\varphi \cdot u \circ f$ is a solution of the heat equation in $D$ whenever $u$ is a solution of the heat equation in $E$. We give the characterization of caloric morphisms, and then give the determination of caloric morphisms. In the case of $m<n$, there are no caloric morphisms. In the case of $m=n$, caloric morphisms are generated by the dilation, the rotation, the translation and the Appell transformation. In the case of $m>n$, under some assumption on $f$, every caloric morphism is obtained by composing a projection with a direct sum of caloric morphisms of $\mathbb{R}^{n+1}$.
\end{abstract}

\section{$\S 1$. Introduction}

For a non-negative integer $k, \mathbb{R}^{k+1}$ denotes the $k+1$-dimensional Euclidean space. The coordinates in $\mathbb{R}^{k+1}$ is denoted by $(t, x)$ or $\left(x_{0}, x\right)$ where $x=\left(x_{1}, \ldots, x_{k}\right)$.

We shall use the following notation:

$$
\nabla=\left(\frac{\partial}{\partial x_{1}}, \ldots, \frac{\partial}{\partial x_{k}}\right), \quad \Delta=\sum_{j=1}^{k} \frac{\partial^{2}}{\partial x_{j}^{2}}, \quad H=\frac{\partial}{\partial t}-\Delta
$$

A $C^{2}$-function $h$ is said to be caloric if $h$ satisfies the heat equation

$$
H h=0 .
$$

Since the heat operator $H$ is hypoelliptic (see, e.g. [9]), every caloric function is infinitely differentiable.

Received November 12, 1998.

2000 Mathematics Subject Classification: 31B99, 35K99, 35A30 
Let $m, n$ be positive integers and $D$ a domain in $\mathbb{R}^{m+1}$. We denote by $(t, x)=\left(t, x_{1}, \ldots, x_{m}\right),(\tau, y)=\left(\tau, y_{1}, \ldots, y_{n}\right)$ the points of $\mathbb{R}^{m+1}, \mathbb{R}^{n+1}$ respectively. We consider a mapping $f(t, x)=\left(f_{0}(t, x), f_{1}(t, x), \ldots, f_{n}(t, x)\right)$ : $D \rightarrow \mathbb{R}^{n+1}$ and a weight function $\varphi$ which preserve solutions of the heat equation in the following sense. A pair $(f, \varphi)$ of $C^{2}$-mapping $f: D \rightarrow \mathbb{R}^{n+1}$ and a positive $C^{2}$-function $\varphi$ on $D$ is said to be a caloric morphism if $f(D)$ is a domain in $\mathbb{R}^{n+1}$ and if for every caloric function $u$ on $f(D)$, $\varphi(t, x)(u \circ f)(t, x)$ is also a caloric function on $D$.

In the case of $m=n$, the following three typical caloric morphisms are known.

\section{The Appell transformation}

Let $D=(0, \infty) \times \mathbb{R}^{n}\left(\right.$ resp. $\left.=(-\infty, 0) \times \mathbb{R}^{n}\right)$. Put

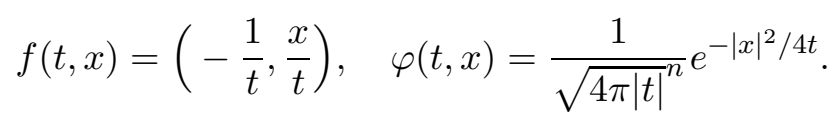

Then $f(D)=(-\infty, 0) \times \mathbb{R}^{n}$ (resp. $\left.=(0, \infty) \times \mathbb{R}^{n}\right)$ and $(f, \varphi)$ is a caloric morphism.

\section{The dilation and the rotation in $x$}

Let $\lambda>0$ and $U$ be an $(n, n)$-orthogonal matrix. Put

$$
f(t, x)=\left(\lambda^{2} t, \lambda U x\right), \quad \varphi(t, x)=1 .
$$

Then $(f, \varphi)$ is a caloric morphism from $\mathbb{R}^{n+1}$ onto $\mathbb{R}^{n+1}$.

\section{The translation}

Let $a \in \mathbb{R}$ and $b, c \in \mathbb{R}^{n}$. Put

$$
f(t, x)=(t+a, x+t b+c), \quad \varphi(t, x)=e^{\frac{1}{4}|b|^{2} t+\frac{1}{2} b \cdot x} .
$$

Then $(f, \varphi)$ is a caloric morphism from $\mathbb{R}^{n+1}$ onto $\mathbb{R}^{n+1}$.

We give two simple examples in the case of $m>n$.

EXAMPLE 1 . The symmetrization in $\mathbb{R}^{m}$ with respect to a subspace with codimension 2 .

Let $m \geqq 4, n=m-2$ and $D=\left\{(t, x) ; t>0,\left|x^{\prime}\right|>0\right\}$ (resp. $\left.D=\left\{(t, x) ; t<0,\left|x^{\prime}\right|>0\right\}\right)$, where $x^{\prime}=\left(x_{1}, x_{2}, x_{3}, 0, \ldots, 0\right)$ for $x=$ $\left(x_{1}, \ldots, x_{m}\right)$. Put

$$
\left\{\begin{array}{l}
f_{0}(t)=-t^{-1} \\
f_{1}(t, x)=t^{-1}\left|x^{\prime}\right|, \\
f_{j}(t, x)=t^{-1} x_{j+2}, \quad 2 \leqq j \leqq n
\end{array}\right.
$$




$$
\varphi(t, x)=\left|x^{\prime}\right|^{-1}|t|^{-(m-2) / 2} \exp \left(-\frac{|x|^{2}}{4 t}\right) .
$$

Then $f(D)=\left\{(\tau, y) ; \tau<0, y_{1}>0\right\}$ (resp. $\left.f(D)=\left\{(\tau, y) ; \tau>0, y_{1}<0\right\}\right)$ and $(f, \varphi)$ is a caloric morphism.

EXAMPLE 2. The projection in $x$.

Let $h$ be an arbitrary positive caloric function on $\mathbb{R}^{m-n+1}$. Put

$$
f\left(t, x_{1}, \ldots, x_{m}\right)=\left(t, x_{1}, \ldots, x_{n}\right), \quad \varphi(t, x)=h\left(t, x_{n+1}, \ldots, x_{m}\right) .
$$

Then $(f, \varphi)$ is a caloric morphism from $\mathbb{R}^{m+1}$ onto $\mathbb{R}^{n+1}$.

In the case of $m=n$, Leutwiler [7] proved that every caloric morphism has the following form:

$$
\begin{aligned}
& f(t, x)=\left(\frac{\alpha t+\beta}{\gamma t+\delta}, \frac{R x+t v+w}{\gamma t+\delta}\right), \\
& \varphi(t, x)= \begin{cases}\frac{C}{|\gamma t+\delta|^{n / 2}} \exp \left[-\frac{|\gamma R x+\gamma w-\delta v|^{2}}{\gamma|\gamma t+\delta|}\right], & \gamma \neq 0, \\
C \exp \left[\frac{|v|^{2}}{4} t+\frac{1}{2} v \cdot R x\right], & \gamma=0,\end{cases}
\end{aligned}
$$

where $\alpha, \beta, \gamma, \delta$ are real numbers with $\alpha \delta-\beta \gamma=1, v, w \in \mathbb{R}^{n}, R$ is an $n$-dimensional orthogonal matrix, $C>0$ and $\cdot$ denotes the inner product of $\mathbb{R}^{n}$. It is a composition of the above three morphisms: the Appell transformation, the dilation, the translation.

The aim of this paper is to extend this to the case of $m \neq n$.

We first give a general characterization of caloric morphisms, which is essentially obtained by Leutwiler. As its corollary, there are no caloric morphism if $m<n$. Also by virtue of the characterization, we obtain a new systematic way to construct a caloric morphism by a "direct sum" of caloric morphisms in the case of $m>n$. It is remarkable that the direct sum gives caloric morphisms of new type such that $f_{0}$ is a sum of fractional linear functions. Note that in the case of $m=n, f_{0}$ is just a fractional linear function.

Our main result is the determination of caloric morphisms $(f, \varphi)$ in the case of $m>n$ under the assumption that each $f_{i}, 1 \leqq i \leqq n$ is a polynomial in $x$ for every $t$ and that $f_{0}$ is real analytic. Under the assumption, we can give an explicit form of caloric morphisms (Theorem 7 below). Although it seems to be complicated, it turns out to be a direct sum of the caloric morphisms of form (0) composed with a projection, as is shown in Corollary 10. 


\section{$\S 2$. Characterization of caloric morphisms}

Definition 1. A pair $(f, \varphi)$ of $C^{2}$-mapping $f: D \rightarrow \mathbb{R}^{n+1}$ and a positive $C^{2}$-function on $D$ is said to be a caloric morphism, if $f(D)$ is a domain and if for every caloric function $u$ on $f(D), \varphi(t, x)(u \circ f)(t, x)$ is also a caloric function on $D$.

Remark 1. Using derivatives in the sense of distribution, we may assume $f$ and $\varphi$ to be continuous rather than of $C^{2}$. For the sake of simplicity, we assume here that $f$ and $\varphi$ are of $C^{2}$.

TheOREm 1. Let $f=\left(f_{0}, f_{1}, \ldots, f_{n}\right): D \rightarrow \mathbb{R}^{n+1}$ be a $C^{2}$-mapping such that $f(D)$ is a domain and let $\varphi$ be a positive $C^{2}$-function on $D$. Then the following statements are equivalent:

(i) $(f, \varphi)$ is a caloric morphism.

(ii) For every polynomial $P(\tau, y)$ which is caloric and of degree $\leqq 4$,

$$
\varphi(t, x)(P \circ f)(t, x)
$$

is caloric on $D$.

(iii) $f$ and $\varphi$ satisfy the following equations:

$$
\begin{aligned}
& H \varphi=0, \\
& \varphi H f_{i}=2 \nabla \varphi \cdot \nabla f_{i}, \quad 1 \leqq i \leqq n, \\
& \nabla f_{0}=0, \\
& \nabla f_{i}(t, x) \cdot \nabla f_{j}(t, x)=\delta_{i j} \frac{d f_{0}}{d t}(t), \quad 1 \leqq i, j \leqq n,
\end{aligned}
$$

where $\cdot$ denotes the inner product in $\mathbb{R}^{m}$.

(iv) There exists a continuous function $\lambda(t) \geqq 0$ on $D$ such that

$$
H\{\varphi(u \circ f)\}(t, x)=\lambda(t)^{2} \varphi(t, x)(H u \circ f)(t, x)
$$

holds for every $C^{2}$ function $u$ on $f(D)$ where $H$ in the right hand side means the heat operator on $\mathbb{R}^{n+1}$.

Remark 2. By (3), $f_{0}$ depends only on $t$. And (4) shows that $d f_{0} / d t \geqq 0$ and $\left|\nabla f_{j}(t, x)\right|^{2}$ is independent of $x$, where $|\cdot|$ denotes the norm of $\mathbb{R}^{m}$. 
Proof.

(i) $\Rightarrow$ (ii) is trivial.

(ii) $\Rightarrow$ (iii): By the chain rule,

$$
\begin{aligned}
H\{\varphi(P \circ f)\}=H \varphi(P \circ f)+ & \sum_{i=0}^{n}\left(\varphi H f_{i}-2 \nabla \varphi \cdot \nabla f_{i}\right) \frac{\partial P}{\partial y_{i}} \circ f \\
& -\varphi \sum_{i, j=0}^{n}\left(\nabla f_{i} \cdot \nabla f_{j}\right) \frac{\partial^{2} P}{\partial y_{i} \partial y_{j}} \circ f .
\end{aligned}
$$

Let $P=1$. Then we have $H \varphi=0$. Let $P\left(y_{0}, y\right)=y_{i}, 1 \leqq i \leqq n$ in the equation (6). Then we obtain

$$
\varphi H f_{i}=2 \nabla \varphi \cdot \nabla f_{i}, \quad 1 \leqq i \leqq n
$$

Take a point $p \in D$ and put $q=f(p)$. Let $P\left(y_{0}, y\right)=\left(y_{i}-q_{i}\right)\left(y_{j}-q_{j}\right)$, $1 \leqq i, j \leqq n, i \neq j$ in the equation (6). Since $\left(\partial^{2} P / \partial y_{i} \partial y_{j}\right)(q)=1$ and the other derivatives of $P$ vanish at $q$, we have

$$
\nabla f_{i}(p) \cdot \nabla f_{j}(p)=0, \quad 1 \leqq i, j \leqq n, i \neq j .
$$

Since $p$ is arbitrary,

$$
\nabla f_{i} \cdot \nabla f_{j}=0, \quad 1 \leqq i, j \leqq n, i \neq j,
$$

in $D$. Let $P\left(y_{0}, y\right)=\left(y_{0}-q_{0}\right)^{2}+\left(y_{0}-q_{0}\right)\left(y_{i}-q_{i}\right)^{2}+\frac{1}{12}\left(y_{i}-q_{i}\right)^{4}, 1 \leqq i \leqq n$. Since $\left(\partial^{2} P / \partial y_{0}^{2}\right)(q)=1$ and the other derivatives of order $\leqq 2$ vanish at $q$, we have

$$
\left|\nabla f_{0}(p)\right|^{2}=0, \text { and thus } \nabla f_{0}(p)=0
$$

Since $p$ is arbitrary, $(3)$ holds. Finally, let $P\left(y_{0}, y\right)=y_{0}-q_{0}+\frac{1}{2}\left(y_{i}-q_{i}\right)^{2}$, $1 \leqq i \leqq n$. Since $\left(\partial P / \partial y_{0}\right)(q)=\left(\partial^{2} P / \partial y_{i}^{2}\right)(q)=1$ and the other derivatives vanish at $q$, we have

$$
\varphi(p) H f_{0}(p)=\varphi(p)\left|\nabla f_{i}(p)\right|^{2}, \quad 1 \leqq i \leqq n
$$

Combining (7), (8) and (9), we obtain (4).

(iii) $\Rightarrow\left(\right.$ iv): Let $u$ be of $C^{2}$ in $f(D)$. By the chain rule

$$
\begin{aligned}
H\{\varphi(u \circ f)\}=H \varphi(u \circ f)+ & \sum_{i=0}^{n}\left(\varphi H f_{i}-2 \nabla \varphi \cdot \nabla f_{i}\right) \frac{\partial u}{\partial y_{i}} \circ f \\
& -\varphi \sum_{i, j=0}^{n}\left(\nabla f_{i} \cdot \nabla f_{j}\right) \frac{\partial^{2} u}{\partial y_{i} \partial y_{j}} \circ f .
\end{aligned}
$$


Substituting (1)-(4) into (10), we have

$$
H\{\varphi(u \circ f)\}=\varphi H f_{0} \frac{\partial u}{\partial y_{0}} \circ f-\varphi \sum_{i=1}^{n}\left|\nabla f_{i}\right|^{2} \frac{\partial^{2} u}{\partial y_{i}^{2}} \circ f=\varphi \frac{d f_{0}}{d t} H u \circ f
$$

Putting $\lambda(t)=\left(d f_{0} / d t(t)\right)^{1 / 2}$, we obtain

$$
H\{\varphi(u \circ f)\}(t, x)=\lambda(t)^{2} \varphi(t, x)(H u \circ f)(t, x) .
$$

Note that $\lambda(t)=\left|\nabla f_{i}(t, x)\right|$ by $(4)$.

(iv) $\Rightarrow$ (i) is evident.

Corollary 2. For every caloric morphism $(f, \varphi), f$ and $\varphi$ are of $C^{\infty}$.

Proof. By (2), $\varphi f_{i}$ is caloric $(1 \leqq i \leqq n)$, so $\varphi f_{i}$ is of $C^{\infty}$. Since $\varphi>0$ and $\varphi$ is caloric, $f_{i}$ is of $C^{\infty}, 1 \leqq i \leqq n$. $f_{0}$ is of $C^{\infty}$ by (4). Thus $f$ is a $C^{\infty}$-mapping.

Corollary 3. Let $(f, \varphi)$ be a caloric morphism from $D$ to $\mathbb{R}^{n+1}$. Then for any $C^{2}$-function $u$ on $f(D)$, we have the following implications:

$$
\begin{aligned}
& H u \geqq 0 \Longrightarrow H\{\varphi(u \circ f)\} \geqq 0, \\
& H u \leqq 0 \Longrightarrow H\{\varphi(u \circ f)\} \leqq 0 .
\end{aligned}
$$

They immediately follow from (5).

Corollary 4. (i) Let $(f, \varphi)=\left(\left(f_{0}, \ldots, f_{n}\right), \varphi\right)$ be a caloric morphism from $D \subset \mathbb{R}^{m+1}$ to $\mathbb{R}^{n+1}$. Then $f_{0}^{\prime}(t)>0$ on $D$.

(ii) If $n>m$, there are no caloric morphisms.

Proof. (i) Suppose that $f_{0}^{\prime}\left(t_{0}\right)=0$ for some $\left(t_{0}, x_{0}\right) \in D$. Let $I \subset \mathbb{R}$ be the connected component of $\left\{t ; f_{0}^{\prime}(t)=0\right\}$ such that $t_{0} \in I$. Since $f_{0}$ is a non-decreasing function, $f_{0}(t) \neq f_{0}\left(t_{0}\right)$ for all $t \notin I$. So we have

$$
f(\{(t, x) \in D ; t \in I\})=f(D) \cap\left\{(\tau, y) \in \mathbb{R}^{n+1} ; \tau=f_{0}\left(t_{0}\right)\right\} .
$$

Then by (4)

$$
\nabla f_{i}(t, x)=0, \quad(t, x) \in D, t \in I, 1 \leqq i \leqq n
$$


This and (2) imply

$$
\frac{\partial f_{i}}{\partial t}(t, x)=2 \nabla \log \varphi \cdot \nabla f_{i}=0, \quad(t, x) \in D, t \in I, 1 \leqq i \leqq n .
$$

Therefore the set $f(\{(t, x) \in D ; t \in I\})$ consists of one point. Thus the set $f(D) \cap\left\{(\tau, y) ; \tau=f_{0}\left(t_{0}\right)\right\}$ consists of one point. It is contrary to the condition that $f(D)$ is a domain. Therefore $f_{0}^{\prime}(t)>0$ for all $t$.

(ii) Let $m<n$. By virtue of (4), $\nabla f_{1}, \ldots, \nabla f_{n}$ are $n$ orthogonal vectors in $\mathbb{R}^{m}$ with same length. Since $n>m$, we have $\nabla f_{1}=\cdots=\nabla f_{n}=0$ in $D$. Then (4) gives $f_{0}^{\prime}=0$ in $D$. This contradicts to (i).

Let $m, n, k$ be positive integers and let $D, E$ be domains in $\mathbb{R}^{m+1}$, in $\mathbb{R}^{n+1}$, respectively. If $(f, \varphi): E \rightarrow \mathbb{R}^{k+1}$ and $(g, \psi): D \rightarrow \mathbb{R}^{n+1}$ are caloric morphisms such that $g(D) \subset E$, then we can make a caloric morphism $(F, \Phi): D \rightarrow \mathbb{R}^{k+1}$ from $(f, \varphi)$ and $(g, \psi)$ by the composition $(F, \Phi)=$ $(f \circ g,(\varphi \circ g) \psi)$.

The next proposition provides a manner for the construction of new caloric morphisms.

Proposition 5. Let $l, m_{1}, \ldots, m_{l}, n$ be positive integers and $I$ be an open interval. For each $j=1, \ldots, l$, suppose that $D_{j}$ is a domain in $\mathbb{R}^{m_{j}}$ and that $\left(g_{j}, \varphi_{j}\right)=\left(\left(g_{j 0}, g_{j 1}, \ldots, g_{j n}\right), \varphi_{j}\right)$ is a caloric morphism $: I \times D_{j} \subset$ $\mathbb{R}^{m_{j}+1} \rightarrow \mathbb{R}^{n+1}$. Put

$$
\begin{aligned}
& f_{0}(t)=g_{10}(t)+\cdots+ \\
& \begin{aligned}
f_{i}\left(t, x_{1}, \ldots, x_{m_{1}+\cdots}(t)\right. & \left.m_{l}\right)=g_{1 i}\left(t, x_{1}, \ldots, x_{m_{1}}\right) \\
& +g_{2 i}\left(t, x_{m_{1}+1}, \ldots, x_{m_{1}+m_{2}}\right)+\cdots \\
& +g_{l i}\left(t, x_{m_{1}+\cdots+m_{l-1}+1}, \ldots, x_{m_{1}+\cdots+m_{l}}\right), \quad 1 \leqq i \leqq n
\end{aligned} \\
& \varphi\left(t, x_{1}, \ldots, x_{m_{1}+\cdots+m_{l}}\right)=\varphi_{1}\left(t, x_{1}, \ldots, x_{m_{1}}\right) \varphi_{2}\left(t, x_{m_{1}+1}, \ldots, x_{m_{1}+m_{2}}\right) \cdots \\
& \varphi_{l}\left(t, x_{m_{1}+\cdots+m_{l-1}+1}, \ldots, x_{m_{1}+\cdots+m_{l}}\right) .
\end{aligned}
$$

Then $(f, \varphi): I \times D_{1} \times \cdots \times D_{l} \subset \mathbb{R}^{m_{1}+\cdots+m_{l}+1} \rightarrow \mathbb{R}^{n+1}$ is a caloric morphism.

We call the above caloric morphism $(f, \varphi)$ the direct sum of $\left(g_{1}, \varphi_{1}\right), \ldots,\left(g_{l}, \varphi_{l}\right)$. 
Proof. For each $j$, we denote by $H_{j}, \nabla_{j}$ and $\Delta_{j}$ the heat operator, the gradient and the Laplacian in $\mathbb{R}^{m_{j}+1}$. The heat operator, the gradient and the Laplacian in $\mathbb{R}^{m_{1}+\cdots+m_{l}+1}$ are denoted by $H, \nabla$ and $\Delta$. Since $\left(g_{j}, \varphi_{j}\right)$ is a caloric morphism, (1), (2) and (4) show

$$
\begin{array}{r}
H_{j} \varphi_{j}=0, \quad \varphi_{j} H_{j} g_{j i}=2 \nabla_{j} \varphi_{j} \cdot \nabla_{j} g_{j i}, \quad \nabla_{j} g_{j i} \cdot \nabla_{j} g_{j k}=\delta_{i k} \frac{d g_{j 0}}{d t}, \\
1 \leqq i, k \leqq n, 1 \leqq j \leqq l .
\end{array}
$$

Using

$$
\begin{aligned}
\nabla f_{i} & =\left(\nabla_{1} g_{1 i}, \nabla_{2} g_{2 i}, \ldots, \nabla_{l} g_{l i}\right), \\
\nabla \varphi & =\varphi\left(\frac{\nabla_{1} \varphi_{1}}{\varphi_{1}}, \frac{\nabla_{2} \varphi_{2}}{\varphi_{2}}, \ldots, \frac{\nabla_{l} \varphi_{l}}{\varphi_{l}}\right), \\
H f_{i} & =H_{1} g_{1 i}+H_{2} g_{2 i}+\cdots+H_{l} g_{l i},
\end{aligned}
$$

we have

$$
\begin{aligned}
2 \nabla \varphi \cdot \nabla f_{i} & =\varphi\left(\frac{2 \nabla_{1} \varphi_{1} \cdot \nabla_{1} g_{1 i}}{\varphi_{1}}, \frac{2 \nabla_{2} \varphi_{2} \cdot \nabla_{2} g_{2 i}}{\varphi_{2}}, \ldots, \frac{2 \nabla_{l} \varphi_{l} \cdot \nabla_{l} g_{l i}}{\varphi_{l}}\right) \\
& =\varphi\left(H_{1} g_{1 i}+H_{2} g_{2 i}+\cdots+H_{l} g_{l i}\right) \\
& =\varphi H f_{i}, \quad 1 \leqq i \leqq n
\end{aligned}
$$

and

$$
\begin{aligned}
\nabla f_{i} \cdot \nabla f_{k} & =\nabla_{1} g_{1 i} \cdot \nabla_{1} g_{1 k}+\nabla_{2} g_{2 i} \cdot \nabla_{2} g_{2 k}+\cdots+\nabla_{l} g_{l i} \cdot \nabla_{l} g_{l k} \\
& =\delta_{i k}\left(\frac{d g_{10}}{d t}+\frac{d g_{20}}{d t}+\cdots+\frac{d g_{l 0}}{d t}\right) \\
& =\delta_{i k} \frac{d f_{0}}{d t}
\end{aligned}
$$

On the other hand, since

$$
\begin{aligned}
& \frac{\partial \varphi}{\partial t}=\varphi\left(\frac{1}{\varphi_{1}} \frac{\partial \varphi_{1}}{\partial t}+\frac{1}{\varphi_{2}} \frac{\partial \varphi_{2}}{\partial t}+\cdots+\frac{1}{\varphi_{l}} \frac{\partial \varphi_{l}}{\partial t}\right) \\
& \Delta \varphi=\varphi\left(\frac{\Delta_{1} \varphi_{1}}{\varphi_{1}}+\frac{\Delta_{2} \varphi_{2}}{\varphi_{2}}+\cdots+\frac{\Delta_{l} \varphi_{l}}{\varphi_{l}}\right)
\end{aligned}
$$

we obtain $H \varphi=0$. Thus $(f, \varphi)$ is a caloric morphism. 


\section{$\S 3$. Main result}

In the case of $m=n$, the form of caloric morphism is explicitly determined by Leutwiler [7]. So hereafter, we assume $m>n$ in the rest of this paper.

In the sequel, we shall determine caloric morphisms $(f, \varphi), f=\left(f_{0}, f_{1}\right.$, $\left.\ldots, f_{n}\right)$ in the case that $f_{i}, 1 \leqq i \leqq n$ is a polynomial of $x$ for each $t$ and that $f_{0}$ is real analytic.

Proposition 6. Let $(f, \varphi)$ be a caloric morphism and assume that $f_{i}, 1 \leqq i \leqq n$ is a polynomial of $x$ for each fixed $t$. Then

$$
f_{i}(t, x)=\sum_{j=1}^{m} a_{i j}(t) x_{j}+b_{i}(t), \quad 1 \leqq i \leqq n,
$$

where $a_{i j}, b_{i}, 1 \leqq i \leqq n, 1 \leqq j \leqq m$ are $C^{\infty}$-functions.

Proof. Let $t$ be fixed. Suppose that $f_{i}(t, x)$ is a polynomial of degree $l \geqq 1$. Write $f_{i}(t, x)=h(t, x)+g(t, x)$, where $h$ is a homogeneous polynomial of degree $l$ and $g$ is a polynomial of degree $\leqq l-1$. Since $\nabla h \neq 0$, the degree of the polynomial $\left|\nabla f_{i}\right|^{2}=|\nabla h|^{2}+2 \nabla h \cdot \nabla g+|\nabla g|^{2}$ is equal to $2 l-2$. On the other hand, $\left|\nabla f_{i}\right|^{2}$ is of degree 0 by (4) of Theorem 1 . Thus $\operatorname{deg} f_{i} \leqq 1$.

Remark 3. We cannot replace real analytic functions in the place of polynomials in the above proposition. In the above Example 1, $f_{1}$ is not a polynomial.

Main result of this paper is the following

TheOREM 7. Let $(f, \varphi)=\left(\left(f_{0}, f_{1}, \ldots, f_{n}\right), \varphi\right)$ be a caloric morphism defined on a domain $D \subset \mathbb{R}^{m+1}$. Assume that for each $1 \leqq i \leqq n$ and each $t, f_{i}(t, x)$ is a polynomial of $x$ and that $f_{0}(t)$ is real analytic.

Then there exist a positive integer $k \leqq m / n$ and an orthogonal coordinate of $\mathbb{R}^{m}$ denoted by $\left(x_{1}, \ldots, x_{m}\right)$ again with four families $\alpha_{i}, 1 \leqq i \leqq k$, $\beta_{i}, 1 \leqq i \leqq k, \delta_{i}, 0 \leqq i \leqq n$ and $\gamma_{i j}, 1 \leqq i \leqq n, 1 \leqq j \leqq k$ of real numbers satisfying $\alpha_{i}>0$ and $\beta_{i} \neq \beta_{j}, i \neq j$, and a positive caloric function $h=h\left(t, x_{k n+1}, \ldots, x_{m}\right)$ (in the case of $m=n k, h$ is a positive constant) such that $f$ and $\varphi$ are of form (I) or (II). 
(I)

$$
\begin{aligned}
& f_{0}(t)=\sum_{j=1}^{k} \frac{\alpha_{j}^{2}}{\beta_{j}-t}+\delta_{0} \\
& f_{i}(t, x)=\sum_{j=1}^{k} \frac{\alpha_{j}}{\beta_{j}-t}\left(x_{(j-1) n+i}+\gamma_{i j}\right)+\delta_{i}, \quad 1 \leqq i \leqq n \\
& \varphi(t, x)=h \prod_{j=1}^{k} \frac{1}{\left|\beta_{j}-t\right|^{n / 2}} \exp \sum_{i=1}^{n} \frac{\left(x_{(j-1) n+i}+\gamma_{i j}\right)^{2}}{4\left(\beta_{j}-t\right)},
\end{aligned}
$$

(II)

$$
\begin{aligned}
& f_{0}(t)=\alpha_{1}^{2} t+\sum_{1<j \leqq k} \frac{\alpha_{j}^{2}}{\beta_{j}-t}+\delta_{0}, \\
& f_{i}(t, x)=\alpha_{1}\left(x_{i}+\gamma_{i 1} t\right)+\sum_{1<j \leqq k} \frac{\alpha_{j}}{\beta_{j}-t}\left(x_{(j-1) n+i}+\gamma_{i j}\right)+\delta_{i}, \quad 1 \leqq i \leqq n \\
& \varphi(t, x)=h \exp \sum_{i=1}^{n}\left[\frac{\gamma_{i 1}^{2}}{4} t+\frac{\gamma_{i 1}}{2} x_{i}\right] \prod_{1<j \leqq k} \frac{1}{\left|\beta_{j}-t\right|^{n / 2}} \exp \sum_{i=1}^{n} \frac{\left(x_{(j-1) n+i}+\gamma_{i j}\right)^{2}}{4\left(\beta_{j}-t\right)} .
\end{aligned}
$$

First we shall prove the assertion of the theorem in the case of $n=1$ under the assumption that $\log \varphi$ is a polynomial of $x$ of degree $\leqq 2$.

LEMMA 8. Let $(f, \varphi)=\left(\left(f_{0}, f_{1}\right), \varphi\right)$ be a caloric morphism from $D \subset$ $\mathbb{R}^{m+1}$ to $\mathbb{R}^{1+1}$. Assume that $f_{1}$ and $\varphi$ are of the following form:

$$
\begin{aligned}
f_{1}(t, x) & =\sum_{j=1}^{m} a_{j}(t) x_{j}+b(t), \\
\varphi(t, x) & =\exp \left(\frac{1}{4} x \cdot U(t) x+v(t) \cdot x+w(t)\right),
\end{aligned}
$$

where $a_{1}, \ldots, a_{m}, b$ and $w$ are $C^{\infty}$-functions, $v$ is a $C^{\infty}$-vector and where $U$ is a symmetric $(m, m)$-matrix of $C^{\infty}$-functions.

Then there exist a positive integer $k \leqq m$ and an orthogonal coordinate of $\mathbb{R}^{m}$ denoted by $\left(x_{1}, \ldots, x_{m}\right)$ again with four families $\alpha_{i}, 1 \leqq i \leqq k, \beta_{i}$, $1 \leqq i \leqq k, \delta_{i}, i=0,1$ and $\gamma_{i}, 1 \leqq i \leqq k$ of real numbers satisfying $\alpha_{i}>0$ and $\beta_{i} \neq \beta_{j}, i \neq j$, and a positive caloric function $h=h\left(t, x_{k+1}, \ldots, x_{m}\right)$ 
(in the case of $m=k, h$ is a positive constant) such that $f$ and $\varphi$ are of form (1) or (2).

(1)

$$
\begin{aligned}
& f_{0}(t)=\sum_{j=1}^{k} \frac{\alpha_{j}^{2}}{\beta_{j}-t}+\delta_{0} \\
& f_{1}(t, x)=\sum_{j=1}^{k} \frac{\alpha_{j}}{\beta_{j}-t}\left(x_{j}+\gamma_{j}\right)+\delta_{1} \\
& \varphi(t, x)=h(t, x) \prod_{j=1}^{k} \frac{1}{\left|\beta_{j}-t\right|^{1 / 2}} \exp \frac{\left(x_{j}+\gamma_{j}\right)^{2}}{4\left(\beta_{j}-t\right)}
\end{aligned}
$$

if $U\left(t_{0}\right)$ is invertible or $a\left(t_{0}\right)$ is orthogonal to the zero-eigenspace of $U\left(t_{0}\right)$ for some $t_{0}$.

$$
\begin{aligned}
& f_{0}(t)=\alpha_{1}^{2} t+\sum_{1<j \leqq k} \frac{\alpha_{j}^{2}}{\beta_{j}-t}+\delta_{0} \\
& f_{1}(t, x)=\alpha_{1}\left(x_{1}+\gamma_{1} t\right)+\sum_{1<j \leqq k} \frac{\alpha_{j}}{\beta_{j}-t}\left(x_{j}+\gamma_{j}\right)+\delta_{1} \\
& \varphi(t, x)=h(t, x) \exp \left[\frac{\gamma_{1}^{2}}{4} t+\frac{\gamma_{1}}{2} x_{1}\right] \prod_{1<j \leqq k} \frac{1}{\left|\beta_{j}-t\right|^{1 / 2}} \exp \frac{\left(x_{j}+\gamma_{j}\right)^{2}}{4\left(\beta_{j}-t\right)},
\end{aligned}
$$

otherwise.

Proof of Lemma 8. We may assume $t_{0}=0$ by some translation of $t$.

Since $(f, \varphi)$ is a caloric morphism, $f_{1}$ and $\log \varphi$ satisfy the equations

$$
\begin{aligned}
& \frac{\partial \log \varphi}{\partial t}-\Delta \log \varphi-|\nabla \log \varphi|^{2}=0, \\
& H f_{1}=2 \nabla \log \varphi \cdot \nabla f_{1},
\end{aligned}
$$

by (1) and (2). Then we have the following differential equations

$$
\begin{aligned}
& U^{\prime}=U^{2}, \quad v^{\prime}=U v, \quad w^{\prime}=\frac{|v|^{2}}{4}+\frac{\operatorname{tr} U}{2}, \\
& a^{\prime}=U a, \quad b^{\prime}=a \cdot v,
\end{aligned}
$$


where $a=\left(a_{1}, \ldots, a_{m}\right)$ and $\operatorname{tr} U$ denotes the trace of the matrix $U$.

Since $U(0)$ is real symmetric, we have the spectral decomposition $U(0)=\sum_{j=1}^{l} \lambda_{j} P_{j}$, where $\lambda_{j}$ is a real eigenvalue of $U(0)$ with multiplicity $n_{j}$, and $P_{j}$ is the orthogonal projection of $\mathbb{R}^{m}$ to the corresponding eigenspace. Since $U(t)$ is the solution of $U^{\prime}=U^{2}$,

$$
U(t)=\sum_{j=1}^{l} \frac{\lambda_{j}}{1-\lambda_{j} t} P_{j},
$$

and so the solutions of $a^{\prime}=U a, v^{\prime}=U v$ are

$$
a(t)=\sum_{j=1}^{l} \frac{1}{1-\lambda_{j} t} P_{j} a_{0}, \quad v(t)=\sum_{j=1}^{l} \frac{1}{1-\lambda_{j} t} P_{j} v_{0},
$$

where $a_{0}=a(0)$ and $v_{0}=v(0)$.

Let $k$ be the cardinal of $\left\{P_{j} ; P_{j} a_{0} \neq 0\right\}$ (note that $a_{0} \neq 0$ because of (4) and Corollary 4). We may assume $P_{j} a_{0} \neq 0,1 \leqq j \leqq k, P_{j} a_{0}=0, k<j \leqq l$ and $\lambda_{j} \neq 0,1<j \leqq k, k+1<j \leqq l$ by some rearrangement of $\lambda_{1}, \ldots, \lambda_{l}$, if necessary.

Assume that $U(0)$ is invertible. Then $\lambda_{j} \neq 0$ for all $j$ and the solutions of $b^{\prime}=a \cdot v$ and $w^{\prime}=|v|^{2} / 4+\operatorname{tr} U / 2$ are

$$
\begin{aligned}
b(t) & =\sum_{j=1}^{k} \frac{P_{j} a_{0} \cdot P_{j} v_{0}}{\lambda_{j}\left(1-\lambda_{j} t\right)}+\delta_{1}, \\
w(t) & =\sum_{j=1}^{l}\left(\frac{\left|P_{j} v_{0}\right|^{2}}{4 \lambda_{j}\left(1-\lambda_{j} t\right)}-\frac{n_{j}}{2} \log \left(1-\lambda_{j} t\right)\right)+\delta_{2}
\end{aligned}
$$

with some constants $\delta_{1}$ and $\delta_{2}$. By $f_{0}^{\prime}=\left|\nabla f_{1}\right|^{2}$ we have

$$
f_{0}(t)=\int|a(t)|^{2} d t=\sum_{j=1}^{k} \frac{\left|P_{j} a_{0}\right|^{2}}{\lambda_{j}\left(1-\lambda_{j} t\right)}+\delta_{0}
$$

with some constant $\delta_{0}$. Put

$$
\alpha_{j}=\frac{\left|P_{j} a_{0}\right|}{\left|\lambda_{j}\right|}>0, \quad e_{j}=\frac{\lambda_{j} P_{j} a_{0}}{\left|\lambda_{j} P_{j} a_{0}\right|} \in \mathbb{R}^{m}, \quad \beta_{j}=\frac{1}{\lambda_{j}}, \quad 1 \leqq j \leqq k .
$$

Note that $\beta_{1}, \ldots, \beta_{k}$ are mutually distinct. Adding $m-k$ eigenvectors of $U(0)$ to $\left\{e_{1}, \ldots, e_{k}\right\}$, in the case of $m>k$, we obtain an orthonormal basis 
$\left\{e_{1}, \ldots, e_{m}\right\}$ of $\mathbb{R}^{m}$. For $j>k$, we denote by $\lambda_{j}$ the eigenvalue of $U(0)$ corresponding to $e_{j}$ and put $\beta_{j}=\frac{1}{\lambda_{j}}$. By the orthogonal coordinate of $\mathbb{R}^{m}$ defined by $\left\{e_{1}, \ldots, e_{m}\right\}$, we write $x=\left(x_{1}, \ldots, x_{m}\right)$ again for every $x \in \mathbb{R}^{m}$. Putting $\gamma_{j}=e_{j} \cdot \sum_{i=1}^{l} P_{i} v_{0} / \lambda_{i}, 1 \leqq j \leqq m$, we obtain

$$
\begin{aligned}
& f_{0}(t)=\sum_{j=1}^{k} \frac{\alpha_{j}^{2}}{\beta_{j}-t}+\delta_{0} \\
& f_{1}(t, x)=\sum_{j=1}^{k} \frac{\alpha_{j}}{\beta_{j}-t}\left(x_{j}+\gamma_{j}\right)+\delta_{1}, \\
& \varphi(t, x)=C \prod_{j=1}^{m} \frac{1}{\left|\beta_{j}-t\right|^{1 / 2}} \exp \frac{\left(x_{j}+\gamma_{j}\right)^{2}}{4\left(\beta_{j}-t\right)},
\end{aligned}
$$

where $C$ is a positive constant.

Put

$$
h(t, x)=C \prod_{k<j \leqq m} \frac{1}{\left|\beta_{j}-t\right|^{1 / 2}} \exp \frac{\left(x_{j}+\gamma_{j}\right)^{2}}{4\left(\beta_{j}-t\right)} .
$$

Then $h=h\left(t, x_{k+1}, \ldots, x_{m}\right)$ is a positive caloric function and

$$
\varphi(t, x)=h \prod_{j=1}^{k} \frac{1}{\left|\beta_{j}-t\right|^{1 / 2}} \exp \frac{\left(x_{j}+\gamma_{j}\right)^{2}}{4\left(\beta_{j}-t\right)} .
$$

Assume that $U(0)$ is not invertible. Then there are two cases: $a_{0}$ is not orthogonal to the zero-eigenspace of $U(0)$, or $a_{0}$ is orthogonal to the zero-eigenspace. They are equivalent to $\lambda_{1}=0$, or $\lambda_{k+1}=0$, respectively.

If $\lambda_{1}=0$, then $b(t), w(t)$ are given by

$$
\begin{aligned}
b(t) & =P_{1} a_{0} \cdot P_{1} v_{0} t+\sum_{1<j \leqq k} \frac{P_{j} a_{0} \cdot P_{j} v_{0}}{\lambda_{j}\left(1-\lambda_{j} t\right)}+\delta_{1}, \\
w(t) & =\frac{\left|P_{1} v_{0}\right|^{2}}{4} t+\sum_{1<j \leqq l}\left(\frac{\left|P_{j} v_{0}\right|^{2}}{4 \lambda_{j}\left(1-\lambda_{j} t\right)}-\frac{n_{j}}{2} \log \left(1-\lambda_{j} t\right)\right)+\delta_{2}
\end{aligned}
$$

with some constants $\delta_{1}$ and $\delta_{2}$. Thus

$$
f_{0}(t)=\left|P_{1} a_{0}\right|^{2} t+\sum_{1<j \leqq k} \frac{\left|P_{j} a_{0}\right|^{2}}{\lambda_{j}\left(1-\lambda_{j} t\right)}+\delta_{0}
$$


with some constant $\delta_{0}$. Put

$$
\begin{aligned}
& \alpha_{j}=\left\{\begin{array}{ll}
\left|P_{j} a_{0}\right|, & j=1, \\
\frac{\left|P_{j} a_{0}\right|}{\left|\lambda_{j}\right|}, & j>1,
\end{array} \quad e_{j}= \begin{cases}\frac{P_{j} a_{0}}{\left|P_{j} a_{0}\right|}, & j=1, \\
\frac{\lambda_{j} P_{j} a_{0}}{\left|\lambda_{j} P_{j} a_{0}\right|}, & j>1,\end{cases} \right. \\
& \beta_{j}=\frac{1}{\lambda_{j}}, \quad 1<j \leqq k .
\end{aligned}
$$

Note that $\beta_{j}$ are mutually distinct. Adding $m-k$ eigenvectors of $U(0)$ to $\left\{e_{1}, \ldots, e_{k}\right\}$, in the case of $m>k$, we obtain an orthonormal basis $\left\{e_{1}, \ldots, e_{m}\right\}$ of $\mathbb{R}^{m}$. If $j>k$ and $U(0) e_{j}=\lambda_{i} e_{j}$ for some $\lambda_{i} \neq 0$, we put $\beta_{j}=1 / \lambda_{i}$. By the orthogonal coordinate of $\mathbb{R}^{m}$ defined by $\left\{e_{1}, \ldots, e_{m}\right\}$, we write $x=\left(x_{1}, \ldots, x_{m}\right)$ again for every $x \in \mathbb{R}^{m}$.

Putting $\gamma_{j}=e_{j} \cdot\left(P_{1} v_{0}+\sum_{1<i \leqq l} P_{i} v_{0} / \lambda_{i}\right), 1 \leqq j \leqq m$, we obtain

$$
\begin{aligned}
& f_{0}(t)=\alpha_{1}^{2} t+\sum_{1<j \leqq k} \frac{\alpha_{j}^{2}}{\beta_{j}-t}+\delta_{0} \\
& f_{1}(t, x)=\alpha_{1}\left(x_{1}+\gamma_{1} t\right)+\sum_{1<j \leqq k} \frac{\alpha_{j}}{\beta_{j}-t}\left(x_{j}+\gamma_{j}\right)+\delta_{1}, \\
& \varphi(t, x)=C \prod_{j \in J_{0}} \exp \left[\frac{\gamma_{j}^{2}}{4} t+\frac{\gamma_{j}}{2} x_{j}\right] \prod_{j \in J_{1}} \frac{1}{\left|\beta_{j}-t\right|^{1 / 2}} \exp \frac{\left(x_{j}+\gamma_{j}\right)^{2}}{4\left(\beta_{j}-t\right)},
\end{aligned}
$$

where $J_{0}=\left\{j ; U(0) e_{j}=0\right\}, J_{1}=\left\{j ; U(0) e_{j} \neq 0\right\}$ and where $C$ is a positive constant.

Put

$$
h(t, x)=C \prod_{\substack{j \in J_{0} \\ k<j \leqq m}} \exp \left[\frac{\gamma_{j}^{2}}{4} t+\frac{\gamma_{j}}{2} x_{j}\right] \prod_{\substack{j \in J_{1} \\ k<j \leqq m}} \frac{1}{\left|\beta_{j}-t\right|^{1 / 2}} \exp \frac{\left(x_{j}+\gamma_{j}\right)^{2}}{4\left(\beta_{j}-t\right)} .
$$

Then $h=h\left(t, x_{k+1}, \ldots, x_{m}\right)$ is a positive caloric function and

$$
\varphi(t, x)=h \exp \left[\frac{\gamma_{1}^{2}}{4} t+\frac{\gamma_{1}}{2} x_{1}\right] \prod_{1<j \leqq k} \frac{1}{\left|\beta_{j}-t\right|^{1 / 2}} \exp \frac{\left(x_{j}+\gamma_{j}\right)^{2}}{4\left(\beta_{j}-t\right)} .
$$

Finally, if $\lambda_{k+1}=0$, then $b(t), w(t)$ are given by

$$
b(t)=\sum_{1 \leqq j \leqq k} \frac{P_{j} a_{0} \cdot P_{j} v_{0}}{\lambda_{j}\left(1-\lambda_{j} t\right)}+\delta_{1}
$$




$$
w(t)=\frac{\left|P_{k+1} v_{0}\right|^{2}}{4} t+\sum_{j \neq k+1}\left(\frac{\left|P_{j} v_{0}\right|^{2}}{4 \lambda_{j}\left(1-\lambda_{j} t\right)}-\frac{n_{j}}{2} \log \left(1-\lambda_{j} t\right)\right)+\delta_{2}
$$

with some constants $\delta_{1}$ and $\delta_{2}$. Thus

$$
f_{0}(t)=\sum_{1 \leqq j \leqq k} \frac{\left|P_{j} a_{0}\right|^{2}}{\lambda_{j}\left(1-\lambda_{j} t\right)}+\delta_{0}
$$

with some constant $\delta_{0}$. Put

$$
\alpha_{j}=\frac{\left|P_{j} a_{0}\right|}{\left|\lambda_{j}\right|}, \quad e_{j}=\frac{\lambda_{j} P_{j} a_{0}}{\left|\lambda_{j} P_{j} a_{0}\right|}, \quad \beta_{j}=\frac{1}{\lambda_{j}}, \quad 1 \leqq j \leqq k .
$$

Note that $\beta_{j}$ are mutually distinct. Adding $m-k$ eigenvectors of $U(0)$ to $\left\{e_{1}, \ldots, e_{k}\right\}$, in the case of $m>k$, we obtain an orthonormal basis $\left\{e_{1}, \ldots, e_{m}\right\}$ of $\mathbb{R}^{m}$. If $j>k$ and $U(0) e_{j}=\lambda_{i} e_{j}$ for some $\lambda_{i} \neq 0$, we put $\beta_{j}=1 / \lambda_{i}$. By the orthogonal coordinate of $\mathbb{R}^{m}$ defined by $\left\{e_{1}, \ldots, e_{m}\right\}$, we write $x=\left(x_{1}, \ldots, x_{m}\right)$ again for every $x \in \mathbb{R}^{m}$.

Putting $\gamma_{j}=e_{j} \cdot\left(P_{k+1} v_{0}+\sum_{1 \leqq i \leqq l, i \neq k+1} P_{i} v_{0} / \lambda_{i}\right), 1 \leqq j \leqq m$, we obtain

$$
\begin{aligned}
& f_{0}(t)=\sum_{j=1}^{k} \frac{\alpha_{j}^{2}}{\beta_{j}-t}+\delta_{0} \\
& f_{1}(t, x)=\sum_{j=1}^{k} \frac{\alpha_{j}}{\beta_{j}-t}\left(x_{j}+\gamma_{j}\right)+\delta_{1} \\
& \varphi(t, x)=C \prod_{j \in J_{0}} \exp \left[\frac{\gamma_{j}^{2}}{4} t+\frac{\gamma_{j}}{2} x_{j}\right] \prod_{j \in J_{1}} \frac{1}{\left|\beta_{j}-t\right|^{1 / 2}} \exp \frac{\left(x_{j}+\gamma_{j}\right)^{2}}{4\left(\beta_{j}-t\right)}
\end{aligned}
$$

where $J_{0}=\left\{j ; U(0) e_{j}=0\right\}, J_{1}=\left\{j ; U(0) e_{j} \neq 0\right\}$ and where $C$ is a positive constant.

Since $1, \ldots, k \in J_{1}$,

$$
h(t, x)=C \prod_{j \in J_{0}} \exp \left[\frac{\gamma_{j}^{2}}{4} t+\frac{\gamma_{j}}{2} x_{j}\right] \prod_{\substack{j \in J_{1} \\ k<j \leqq m}} \frac{1}{\left|\beta_{j}-t\right|^{1 / 2}} \exp \frac{\left(x_{j}+\gamma_{j}\right)^{2}}{4\left(\beta_{j}-t\right)} .
$$

is a positive caloric function and

$$
\varphi(t, x)=h \prod_{1 \leqq j \leqq k} \frac{1}{\left|\beta_{j}-t\right|^{1 / 2}} \exp \frac{\left(x_{j}+\gamma_{j}\right)^{2}}{4\left(\beta_{j}-t\right)} .
$$


For the proof of Theorem 7, we may assume that $f$ is a caloric morphism of the form

$$
f_{i}(t, x)=\sum_{j=1}^{m} a_{i j}(t) x_{j}+b_{i}(t), \quad 1 \leqq i \leqq n,
$$

by virtue of Proposition 6 . Denote by $a_{i}(t)$ the row-vector $\left(a_{i 1}(t), \ldots, a_{i m}(t)\right)$.

We introduce the functions $p_{k}(t), q_{k}(t), k \geqq 1$ which will be used in the proof of Theorem 7 . We define $p_{1}(t)$ and $q_{1}(t)$ by

$$
p_{1}(t)=\frac{f_{0}^{\prime \prime}(t)}{2 f_{0}^{\prime}(t)}, \quad q_{1}(t)=\frac{1}{\sqrt{3}}\left(p_{1}^{\prime}(t)-p_{1}(t)^{2}\right)^{1 / 2} .
$$

(Recall that $f_{0}^{\prime}(t)>0$ for all $t$ by virtue of Corollary 4 ). For $k \geqq 2$, we define $p_{k}(t)$ and $q_{k}(t)$ inductively by

$$
\begin{aligned}
& p_{k}(t)=\frac{q_{k-1}^{\prime}(t)}{k q_{k-1}(t)}+\frac{k-2}{k} p_{k-1}(t) \\
& q_{k}(t)=\frac{k}{\sqrt{2 k+1}}\left(p_{k}^{\prime}(t)-p_{k}^{2}(t)+\frac{2 k-3}{(k-1)^{2}} q_{k-1}^{2}(t)\right)^{1 / 2}
\end{aligned}
$$

if $q_{k-1}(t) \neq 0$. We put $r_{i}(t) \in \mathbb{R}^{m}, 1 \leqq i \leqq n$ by

$$
r_{i}(t)=\frac{1}{\left|a_{i}(t)\right|} a_{i}(t)
$$

(Note that $\left|a_{i}(t)\right|=\sqrt{f_{0}^{\prime}(t)}>0$ for all $i$ and $t$ because of (4)). And we put $r_{n+1}(t), \ldots, r_{k n}(t)$ inductively by

(14) $r_{i+n}(t)= \begin{cases}\frac{1}{q_{1}(t)} r_{i}^{\prime}(t), & 1 \leqq i \leqq n, \\ \frac{1}{q_{j}(t)}\left(r_{i}^{\prime}(t)+q_{j-1}(t) r_{i-n}(t)\right), & (j-1) n+1 \leqq i \leqq j n, 2 \leqq j \leqq k-1,\end{cases}$

if $q_{j}(t) \neq 0,1 \leqq j \leqq k-1$.

The following is the key lemma to prove Theorem 7 .

Lemma 9. Let $l$ be a positive integer. Assume that $q_{1}, \ldots, q_{l}$ are defined on an open interval $I \subset \mathbb{R}$. Then the following statements hold.

(i) If $q_{l} \neq 0$ on $I$, then $r_{1}(t), \ldots, r_{(l+1) n}(t)$ defined in (14) are orthonormal $C^{\infty}$-vectors of $\mathbb{R}^{m}$. Adding arbitrary $C^{\infty}$-vectors $r_{(l+1) n+1}(t), \ldots, r_{m}(t)$ 
such that $\left\{r_{1}(t), \ldots, r_{m}(t)\right\}$ forms an orthonormal basis of $\mathbb{R}^{m}$ for each $t \in I$, in the case of $m \geqq(l+1) n+1$, we take the change of variables

$$
\left\{\begin{array}{l}
\tau=t \\
\xi_{j}=r_{j}(t) \cdot x, \quad 1 \leqq j \leqq m
\end{array}\right.
$$

on $D \cap\left(I \times \mathbb{R}^{m}\right)$. Then there exists a $C^{\infty}$-function $\psi_{l+1}\left(\tau, \xi_{l n+1}, \ldots, \xi_{m}\right)$ on $D \cap\left(I \times \mathbb{R}^{m}\right)$ such that

$$
\begin{gathered}
\log \varphi(\tau, \xi)=\sum_{k=1}^{l}\left(\sum_{i=(k-1) n+1}^{k n} \frac{1}{4} p_{k}(\tau) \xi_{i}^{2}+\frac{1}{2 k} q_{k}(\tau) \xi_{i} \xi_{i+n}+\beta_{i}(\tau) \xi_{i}+\rho_{i}(\tau)\right) \\
\quad+\psi_{l+1}\left(\tau, \xi_{l n+1}, \ldots, \xi_{m}\right), \\
\frac{\partial \psi_{l+1}}{\partial \xi_{i}}=\frac{1}{2} p_{l+1}(\tau) \xi_{i}+\frac{1}{2(l+1)} \sum_{j=l n+1}^{m}\left(r_{i}^{\prime}(\tau) \cdot r_{j}(\tau)\right) \xi_{j}+\beta_{i}(\tau), \\
l n+1 \leqq i \leqq(l+1) n,
\end{gathered}
$$

and

$$
\begin{aligned}
\frac{\partial \psi_{l+1}}{\partial \tau}-\Delta_{\xi} \psi_{l+1} & -\sum_{k=l n+1}^{m} \frac{\partial \psi_{l+1}}{\partial \xi_{k}}\left(\frac{\partial \psi_{l+1}}{\partial \xi_{k}}-\sum_{j=l n+1}^{m}\left(r_{k}^{\prime}(\tau) \cdot r_{j}(\tau)\right) \xi_{j}\right) \\
& +\sum_{i=l n+1}^{(l+1) n}\left(\frac{2 l-1}{4 l^{2}} q_{l}(\tau)^{2} \xi_{i}^{2}+\frac{l-1}{l} q_{l}(\tau) \beta_{i-n}(\tau) \xi_{i}\right)=0
\end{aligned}
$$

where

$$
\beta_{i}= \begin{cases}\frac{b_{i}^{\prime}}{2 \sqrt{f_{0}^{\prime}}}, & 1 \leqq i \leqq n, \\ \frac{1}{2 q_{1}}\left(\beta_{i-n}^{\prime}-p_{1} \beta_{i-n}\right), & n+1 \leqq i \leqq 2 n, \\ \frac{k}{(k+1) q_{k}}\left(\beta_{i-n}^{\prime}-p_{k} \beta_{i-n}+\frac{k-2}{k-1} q_{k-1} \beta_{i-2 n}\right), & \\ k n+1 \leqq i \leqq(k+1) n, 2 \leqq k \leqq l,\end{cases}
$$

and

$$
\begin{aligned}
\rho_{i}(\tau)=\int\left(\frac{1}{2} p_{k}(\tau)+\beta_{i}^{2}(\tau)\right) d \tau & \\
& (k-1) n+1 \leqq i \leqq k n, 1 \leqq k \leqq l
\end{aligned}
$$


(ii) If $q_{l}(t)=0$ for all $t \in I$, then $r_{1}(t), \ldots, r_{l n}(t)$ defined in (14) are orthonormal $C^{\infty}$-vectors of $\mathbb{R}^{m}$ and satisfies the equations

$$
r_{(l-1) n+i}^{\prime}(t)=\left\{\begin{array}{ll}
0, & \text { if } l=1, \\
-q_{l-1}(t) r_{(l-2) n+i}(t), & \text { if } l \geqq 2,
\end{array} \quad 1 \leqq i \leqq n,\right.
$$

for all $t \in I$. Add arbitrary $C^{\infty}$-vectors $r_{l n+1}(t), \ldots, r_{m}(t)$ such that $\left\{r_{1}(t), \ldots, r_{m}(t)\right\}$ forms an orthonormal basis of $\mathbb{R}^{m}$ for each $t \in I$, if necessary. We take the change of variables $(t, x) \mapsto(\tau, \xi)$ defined in $(1)$. Then there exists a $C^{\infty}$-function $\psi_{l+1}\left(\tau, \xi_{l n+1}, \ldots, \xi_{m}\right)$ on $D \cap\left(I \times \mathbb{R}^{m}\right)$ such that (17) $\log \varphi(\tau, \xi)$

$$
\begin{aligned}
& =\sum_{k=1}^{l-1}\left(\sum_{i=(k-1) n+1}^{k n} \frac{1}{4} p_{k}(\tau) \xi_{i}^{2}+\frac{1}{2 k} q_{k}(\tau) \xi_{i} \xi_{i+n}+\beta_{i}(\tau) \xi_{i}+\rho_{i}(\tau)\right) \\
& \quad+\sum_{i=(l-1) n+1}^{l n}\left(\frac{1}{4} p_{l}(\tau) \xi_{i}^{2}+\beta_{i}(\tau) \xi_{i}+\rho_{i}(\tau)\right) \\
& \quad+\psi_{l+1}\left(\tau, \xi_{l n+1}, \ldots, \xi_{m}\right)
\end{aligned}
$$

and

(18) $\frac{\partial \psi_{l+1}}{\partial \tau}-\Delta_{\xi} \psi_{l+1}-\left|\nabla_{\xi} \psi_{l+1}\right|^{2}+\sum_{j, k=l n+1}^{m}\left(r_{k}^{\prime}(\tau) \cdot r_{j}(\tau)\right) \xi_{j} \frac{\partial \psi_{l+1}}{\partial \xi_{k}}=0$

where $\beta_{i}$ and $\rho_{i}, 1 \leqq i \leqq l n$ are defined in $(\mathrm{i})$.

Proof. We shall show the lemma by induction.

First we shall deal with the case of $l=1$. By (4) and Corollary 4,

$$
a_{i}(t) \cdot a_{j}(t)=\nabla f_{i}(t, x) \cdot \nabla f_{j}(t, x)=\delta_{i j} f_{0}^{\prime}(t)>0, \quad 1 \leqq i \leqq n,
$$

which shows that $\left\{r_{1}(t), \ldots, r_{n}(t)\right\}$ is an orthonormal system of $\mathbb{R}^{m}$ for each $t$. Let $r_{n+1}(t), \ldots, r_{m}(t)$ be $m-n$ orthonormal $C^{\infty}$-vectors such that $\left\{r_{1}(t), \ldots, r_{m}(t)\right\}$ is an orthonormal basis of $\mathbb{R}^{m}$. By the chain rule,

$$
\begin{aligned}
\frac{\partial}{\partial t} & =\frac{\partial \tau}{\partial t} \frac{\partial}{\partial \tau}+\sum_{j=1}^{m} \frac{\partial \xi_{j}}{\partial t} \frac{\partial}{\partial \xi_{j}}=\frac{\partial}{\partial \tau}+\sum_{j=1}^{m} r_{j}^{\prime}(\tau) \cdot x \frac{\partial}{\partial \xi_{j}} \\
& =\frac{\partial}{\partial \tau}+\sum_{j, k=1}^{m}\left(r_{j}^{\prime}(\tau) \cdot r_{k}(\tau)\right) \xi_{k} \frac{\partial}{\partial \xi_{j}} \\
\frac{\partial}{\partial x_{i}} & =\frac{\partial \tau}{\partial x_{i}} \frac{\partial}{\partial \tau}+\sum_{j=1}^{m} \frac{\partial \xi_{j}}{\partial x_{i}} \frac{\partial}{\partial \xi_{j}}=\sum_{j=1}^{m} r_{j i}(\tau) \frac{\partial}{\partial \xi_{j}}
\end{aligned}
$$


where $r_{i}(\tau)=\left(r_{i 1}(\tau), \ldots, r_{i m}(\tau)\right), 1 \leqq i \leqq m$. Since $r_{1}(\tau), \ldots, r_{m}(\tau)$ is orthonormal, we have

$$
\begin{aligned}
& \Delta_{x}=\Delta_{\xi}, \\
& \nabla_{x} u \cdot \nabla_{x} v=\nabla_{\xi} u \cdot \nabla_{\xi} v .
\end{aligned}
$$

Since $(f, \varphi)$ is a caloric morphism, Theorem $1(2)$ and Proposition 6 imply

$$
2 \nabla \log \varphi \cdot \nabla f_{i}=\frac{\partial f_{i}}{\partial t}, \quad 1 \leqq i \leqq n .
$$

By (11) we have

$$
f_{i}(\tau, \xi)=\sqrt{f_{0}^{\prime}(\tau)} \xi_{i}+b_{i}(\tau)
$$

and hence

$$
H f_{i}=\frac{\partial f_{i}}{\partial t}=\frac{f_{0}^{\prime \prime}(\tau)}{2 \sqrt{f_{0}^{\prime}(\tau)}} \xi_{i}+\sqrt{f_{0}^{\prime}(\tau)} \sum_{j=1}^{m}\left(r_{i}^{\prime}(\tau) \cdot r_{j}(\tau)\right) \xi_{j}+b_{i}^{\prime}(\tau) .
$$

Then (19) becomes

$$
\frac{\partial \log \varphi}{\partial \xi_{i}}=\frac{1}{2} p_{1}(\tau) \xi_{i}+\frac{1}{2} \sum_{j=1}^{m}\left(r_{i}^{\prime}(\tau) \cdot r_{j}(\tau)\right) \xi_{j}+\beta_{i}(\tau)
$$

Hence we have

$$
r_{i}^{\prime}(\tau) \cdot r_{j}(\tau)=r_{i}(\tau) \cdot r_{j}^{\prime}(\tau), \quad 1 \leqq i, j \leqq n,
$$

because $\left(\partial / \partial \xi_{j}\right)\left(\partial \log \varphi / \partial \xi_{i}\right)=r_{i}^{\prime}(\tau) \cdot r_{j}(\tau)$. On the other hand, $r_{i}(\tau)$. $r_{j}(\tau)=\delta_{i j}$ implies

$$
r_{i}^{\prime}(\tau) \cdot r_{j}(\tau)=-r_{i}(\tau) \cdot r_{j}^{\prime}(\tau), \quad 1 \leqq i, j \leqq m .
$$

Therefore

$$
r_{i}^{\prime}(\tau) \cdot r_{j}(\tau)=0, \quad 1 \leqq i, j \leqq n .
$$

Then by (21) and (24),

$$
\psi_{2}=\log \varphi-\sum_{i=1}^{n}\left(\frac{1}{4} p_{1}(\tau) \xi_{i}^{2}+\frac{1}{2} \sum_{j=n+1}^{m}\left(r_{i}^{\prime}(\tau) \cdot r_{j}(\tau)\right) \xi_{i} \xi_{j}+\beta_{i}(\tau) \xi_{i}+\rho_{i}(\tau)\right)
$$


is a $C^{\infty}$-function of $\tau, \xi_{n+1}, \ldots, \xi_{m}$. Thus we have

(25) $\log \varphi(\tau, \xi)$

$$
\begin{aligned}
= & \sum_{i=1}^{n}\left(\frac{1}{4} p_{1}(\tau) \xi_{i}^{2}+\frac{1}{2} \sum_{j=n+1}^{m}\left(r_{i}^{\prime}(\tau) \cdot r_{j}(\tau)\right) \xi_{i} \xi_{j}+\beta_{i}(\tau) \xi_{i}+\rho_{i}(\tau)\right) \\
& +\psi_{2}\left(\tau, \xi_{n+1}, \ldots, \xi_{m}\right)
\end{aligned}
$$

On the other hand, $\psi_{1}:=\log \varphi$ satisfies

$$
\frac{\partial \psi_{1}}{\partial t}-\Delta \psi_{1}-\left|\nabla \psi_{1}\right|^{2}=0
$$

because $\varphi$ is a positive caloric function. In the coordinate $\left(\tau, \xi_{1}, \ldots, \xi_{m}\right)$, the above equation is

$$
\frac{\partial \psi_{1}}{\partial \tau}+\sum_{j, k=1}^{m}\left(r_{j}^{\prime}(\tau) \cdot r_{k}(\tau)\right) \xi_{k} \frac{\partial \psi_{1}}{\partial \xi_{j}}-\Delta_{\xi} \psi_{1}-\left|\nabla_{\xi} \psi_{1}\right|^{2}=0
$$

Then from (25), we have

$\frac{\partial \psi_{1}}{\partial \tau}=\sum_{i=1}^{n}\left(\frac{1}{4} p_{1}^{\prime}(\tau) \xi_{i}^{2}+\frac{1}{2} \sum_{j=n+1}^{m}\left(r_{i}^{\prime}(\tau) \cdot r_{j}(\tau)\right)^{\prime} \xi_{i} \xi_{j}+\beta_{i}^{\prime}(\tau) \xi_{i}+\rho_{i}^{\prime}(\tau)\right)+\frac{\partial \psi_{2}}{\partial \tau}$

$\frac{\partial \psi_{1}}{\partial \xi_{k}}= \begin{cases}\frac{1}{2} p_{1}(\tau) \xi_{k}+\frac{1}{2} \sum_{j=n+1}^{m}\left(r_{k}^{\prime}(\tau) \cdot r_{j}(\tau)\right) \xi_{j}+\beta_{k}(\tau), & 1 \leqq k \leqq n \\ \frac{1}{2} \sum_{i=1}^{n}\left(r_{i}^{\prime}(\tau) \cdot r_{k}(\tau)\right) \xi_{i}+\frac{\partial \psi_{2}}{\partial \xi_{k}}, & n+1 \leqq k \leqq m\end{cases}$

$\Delta_{\xi} \psi_{1}=\frac{n}{2} p_{1}(\tau)+\Delta_{\xi} \psi_{2}$

Substituting these into (26) and comparing the coefficients with respect to $\xi_{1}, \ldots, \xi_{n}$, we obtain the following:

$$
\begin{array}{r}
\frac{1}{4}\left(p_{1}^{\prime}(\tau)-p_{1}^{2}(\tau)\right) \delta_{i j}-\frac{3}{4} \sum_{k=n+1}^{m}\left(r_{i}^{\prime}(\tau) \cdot r_{k}(\tau)\right)\left(r_{j}^{\prime}(\tau) \cdot r_{k}(\tau)\right)=0 \\
1 \leqq i, j \leqq n
\end{array}
$$

(28) $\frac{1}{2} \sum_{j=n+1}^{m}\left(r_{i}^{\prime}(\tau) \cdot r_{j}(\tau)\right)^{\prime} \xi_{j}+\left(\beta_{i}^{\prime}(\tau)-p_{1}(\tau) \beta_{i}(\tau)\right)$ 


$$
\begin{aligned}
& -2 \sum_{k=n+1}^{m}\left(r_{i}^{\prime}(\tau) \cdot r_{k}(\tau)\right) \frac{\partial \psi_{2}}{\partial \xi_{k}} \\
& +\frac{1}{2} \sum_{j, k=n+1}^{m}\left(r_{i}^{\prime}(\tau) \cdot r_{k}(\tau)\right)\left(r_{k}^{\prime}(\tau) \cdot r_{j}(\tau)\right) \xi_{j}=0, \quad 1 \leqq i \leqq n,
\end{aligned}
$$

and

$$
\text { (29) } \begin{aligned}
\frac{\partial \psi_{2}}{\partial \tau}-\Delta_{\xi} \psi_{2} & -\sum_{k=n+1}^{m} \frac{\partial \psi_{2}}{\partial \xi_{k}}\left(\frac{\partial \psi_{2}}{\partial \xi_{k}}-\sum_{j=n+1}^{m}\left(r_{k}^{\prime}(\tau) \cdot r_{j}(\tau)\right) \xi_{j}\right) \\
+ & \frac{1}{4} \sum_{i=1}^{n} \sum_{j, k=n+1}^{m}\left(r_{i}^{\prime}(\tau) \cdot r_{j}(\tau)\right)\left(r_{i}^{\prime}(\tau) \cdot r_{k}(\tau)\right) \xi_{j} \xi_{k}=0
\end{aligned}
$$

Since $r_{i}^{\prime}(\tau) \cdot r_{j}(\tau)=0,1 \leqq i, j \leqq n, r_{i}^{\prime}(\tau)=\sum_{k=n+1}^{m}\left(r_{i}^{\prime}(\tau) \cdot r_{k}(\tau)\right) r_{k}(\tau)$ for $1 \leqq i \leqq n$. Hence $(27)$ gives

$$
r_{i}^{\prime}(\tau) \cdot r_{j}^{\prime}(\tau)=q_{1}(\tau)^{2} \delta_{i j}, \quad 1 \leqq i, j \leqq n
$$

(Note that $q_{1}(\tau)^{2}=\left|r_{i}^{\prime}(\tau)\right|^{2} \geqq 0$.)

If $q_{1} \neq 0$ on an open interval $I$, then $(24)$ and (30) show that $r_{1}(\tau), \ldots$, $r_{n}(\tau), r_{1}^{\prime}(\tau), \ldots, r_{n}^{\prime}(\tau)$ are linearly independent for all $\tau \in I$. Therefore $m \geqq$ 2n. Putting

$$
r_{i+n}(\tau)=\frac{r_{i}^{\prime}(\tau)}{q_{1}(\tau)}, \quad 1 \leqq i \leqq n,
$$

we have an orthonormal system $\left\{r_{1}(\tau), \ldots, r_{2 n}(\tau)\right\}$ of $\mathbb{R}^{m}$. Adding $m-2 n$ $C^{\infty}$-vectors $r_{2 n+1}(\tau), \ldots, r_{m}(\tau)$ if $m \geqq 2 n+1$, we obtain an orthonormal basis $\left\{r_{1}(\tau), \ldots, r_{m}(\tau)\right\}$ of $\mathbb{R}^{m}$. Then

$$
r_{i}^{\prime}(\tau) \cdot r_{j}(\tau)=q_{1}(\tau) r_{i+n}(\tau) \cdot r_{j}(\tau)=q_{1}(\tau) \delta_{i+n, j}, \quad 1 \leqq i \leqq n, n+1 \leqq j \leqq m
$$

By (25), (28) and (29)

$$
\begin{aligned}
\log \varphi(\tau, \xi)=\sum_{i=1}^{n} & \left(\frac{1}{4} p_{1}(\tau) \xi_{i}^{2}+\frac{1}{2} q_{1}(\tau) \xi_{i} \xi_{i+n}+\beta_{i}(\tau) \xi_{i}+\rho_{i}(\tau)\right) \\
& +\psi_{2}\left(\tau, \xi_{n+1}, \ldots, \xi_{m}\right)
\end{aligned}
$$




$$
\begin{aligned}
\frac{1}{2} q_{1}^{\prime}(\tau) \xi_{i+n} & +\beta_{i}^{\prime}(\tau)-p_{1}(\tau) \beta_{i}(\tau)-2 q_{1}(\tau) \frac{\partial \psi_{2}}{\partial \xi_{i+n}} \\
+ & \frac{1}{2} q_{1}(\tau) \sum_{j=n+1}^{m}\left(r_{i+n}^{\prime}(\tau) \cdot r_{j}(\tau)\right) \xi_{j}=0, \quad 1 \leqq i \leqq n,
\end{aligned}
$$

and

$$
\begin{gathered}
\frac{\partial \psi_{2}}{\partial \tau}-\Delta_{\xi} \psi_{2}-\sum_{k=n+1}^{m} \frac{\partial \psi_{2}}{\partial \xi_{k}}\left(\frac{\partial \psi_{2}}{\partial \xi_{k}}-\sum_{j=n+1}^{m}\left(r_{k}^{\prime}(\tau) \cdot r_{j}(\tau)\right) \xi_{j}\right) \\
+\frac{1}{4} q_{1}(\tau)^{2} \sum_{i=1}^{n} \xi_{i+n}^{2}=0 .
\end{gathered}
$$

If $q_{1}(\tau)=0$ for all $\tau \in I$, then by $(30), r_{i}^{\prime}=0,1 \leqq i \leqq n$ on $I$ so that

$$
\log \varphi(\tau, \xi)=\sum_{i=1}^{n}\left(\frac{1}{4} p_{1}(\tau) \xi_{i}^{2}+\beta_{i}(\tau) \xi_{i}+\rho_{i}(\tau)\right)+\psi_{2}\left(\tau, \xi_{n+1}, \ldots, \xi_{m}\right)
$$

and

$$
\frac{\partial \psi_{2}}{\partial \tau}-\Delta_{\xi} \psi_{2}-\left|\nabla_{\xi} \psi_{2}\right|^{2}+\sum_{j, k=n+1}^{m}\left(r_{k}^{\prime}(\tau) \cdot r_{j}(\tau)\right) \xi_{j} \frac{\partial \psi_{2}}{\partial \xi_{k}}=0
$$

Thus the assertion in the case of $l=1$ is shown.

Assume $l \geqq 2$ and that the assertion for $1, \ldots, l-1$ holds. Suppose that $q_{1} \neq 0, \ldots, q_{l-1} \neq 0$ on some open interval $I$. Then $q_{l}$ is defined on $I$ and $r_{1}(\tau), \ldots, r_{l n}(\tau)$ defined in (14) are orthonormal $C^{\infty}$-vectors on $\mathbb{R}^{m}$. By the assumption on $1, \ldots, l-1$, there exists a $C^{\infty}$-function $\psi_{l}\left(\tau, \xi_{(l-1) n+1}, \ldots, \xi_{m}\right)$ such that

(31) $\log \varphi(\tau, \xi)$

$$
\begin{aligned}
& =\sum_{k=1}^{l-1}\left(\sum_{i=(k-1) n+1}^{k n} \frac{1}{4} p_{k}(\tau) \xi_{i}^{2}+\frac{1}{2 k} q_{k}(\tau) \xi_{i} \xi_{i+n}+\beta_{i}(\tau) \xi_{i}+\rho_{i}(\tau)\right) \\
& \quad+\psi_{l}\left(\tau, \xi_{(l-1) n+1}, \ldots, \xi_{m}\right)
\end{aligned}
$$

$$
\begin{array}{r}
\frac{\partial \psi_{l}}{\partial \xi_{i}}=\frac{1}{2} p_{l}(\tau) \xi_{i}+\frac{1}{2 l} \sum_{j=(l-1) n+1}^{m}\left(r_{i}^{\prime}(\tau) \cdot r_{j}(\tau)\right) \xi_{j}+\beta_{i}(\tau) \\
(l-1) n+1 \leqq i \leqq l n
\end{array}
$$


and

$$
\begin{aligned}
\text { (33) } \frac{\partial \psi_{l}}{\partial \tau}-\Delta_{\xi} \psi_{l}-\sum_{k=(l-1) n+1}^{m} \frac{\partial \psi_{l}}{\partial \xi_{k}}\left(\frac{\partial \psi_{l}}{\partial \xi_{k}}-\sum_{j=(l-1) n+1}^{m}\left(r_{k}^{\prime}(\tau) \cdot r_{j}(\tau)\right) \xi_{j}\right) \\
+\sum_{i=(l-1) n+1}^{l n}\left(\frac{2 l-3}{4(l-1)^{2}} q_{l-1}(\tau)^{2} \xi_{i}^{2}+\frac{l-2}{l-1} q_{l-1}(\tau) \beta_{i-n}(\tau) \xi_{i}\right)=0
\end{aligned}
$$

By (23) and (32)

$$
r_{i}^{\prime}(\tau) \cdot r_{j}(\tau)=0, \quad(l-1) n+1 \leqq i, j \leqq l n
$$

for $\tau \in I$. Put

(35) $\quad \psi_{l+1}$

$$
=\psi_{l}-\sum_{i=(l-1) n+1}^{l n}\left(\frac{1}{4} p_{l}(\tau) \xi_{i}^{2}-\frac{1}{2 l} \sum_{j=l n+1}^{m}\left(r_{i}^{\prime}(\tau) \cdot r_{j}(\tau)\right) \xi_{i} \xi_{j}+\beta_{i}(\tau) \xi_{i}+\rho_{i}(\tau)\right) .
$$

Then $\psi_{l+1}$ is a $C^{\infty}$-function of $\tau, \xi_{l n+1}, \ldots, \xi_{m}$ (in the case of $m=l n$, we have $(1 / 2 l) \sum_{j=l n+1}^{m}\left(r_{i}^{\prime}(\tau) \cdot r_{j}(\tau)\right) \xi_{j}=0$ and $\psi_{l+1}$ depends only on $\left.\tau\right)$. From (35) follow

$$
\begin{aligned}
\frac{\partial \psi_{l}}{\partial \tau}= & \sum_{i=(l-1) n+1}^{l n}\left(\frac{1}{4} p_{l}^{\prime}(\tau) \xi_{i}^{2}+\frac{1}{2 l} \sum_{j=l n+1}^{m}\left(r_{i}^{\prime}(\tau) \cdot r_{j}(\tau)\right)^{\prime} \xi_{i} \xi_{j}+\beta_{i}^{\prime}(\tau) \xi_{i}+\rho_{i}^{\prime}(\tau)\right) \\
& +\frac{\partial \psi_{l+1}}{\partial \tau}, \\
\frac{\partial \psi_{l}}{\partial \xi_{k}}= & \left\{\begin{array}{c}
\frac{1}{2} p_{l}(\tau) \xi_{k}+\frac{1}{2 l} \sum_{j=l n+1}^{m}\left(r_{k}^{\prime}(\tau) \cdot r_{j}(\tau)\right) \xi_{j}+\beta_{k}(\tau), \\
\left.\frac{1}{2 l} \sum_{i=(l-1) n+1}^{l n}\left(r_{i}^{\prime}(\tau) \cdot r_{k}(\tau)\right) \xi_{i}+\frac{\partial \psi_{l+1}}{\partial \xi_{k}}, \quad l n+1\right) n+1 \leqq k \leqq l n
\end{array}\right.
\end{aligned}
$$




$$
\begin{aligned}
& \frac{\partial \psi_{l}}{\partial \xi_{k}}-\sum_{j=(l-1) n+1}^{m}\left(r_{k}^{\prime}(\tau) \cdot r_{j}(\tau)\right) \xi_{j} \\
& =\left\{\begin{array}{cc}
\frac{1}{2} p_{l}(\tau) \xi_{k}-\frac{2 l-1}{2 l} \sum_{j=l n+1}^{m}\left(r_{k}^{\prime}(\tau) \cdot r_{j}(\tau)\right) \xi_{j}+\beta_{k}(\tau), \\
\frac{2 l+1}{2 l} \sum_{i=(l-1) n+1}^{l n}\left(r_{i}^{\prime}(\tau) \cdot r_{k}(\tau)\right) \xi_{i}-\sum_{j=l n+1}^{m}\left(r_{k}^{\prime}(\tau) \cdot r_{j}(\tau)\right) \xi_{j}+\frac{\partial \psi_{l+1}}{\partial \xi_{k}}
\end{array}\right]
\end{aligned}
$$

and

$$
\Delta_{\xi} \psi_{l}=\frac{n}{2} p_{l}(\tau)+\Delta_{\xi} \psi_{l+1}
$$

Substituting these into (33) and comparing the coefficients with respect to $\xi_{(l-1) n+1}, \ldots, \xi_{l n}$, we obtain the following:

$$
\begin{aligned}
& \text { (36) } \frac{1}{4}\left(p_{l}^{\prime}(\tau)-p_{l}(\tau)^{2}+\frac{2 l-3}{(l-1)^{2}} q_{l-1}(\tau)^{2}\right) \delta_{i j} \\
& \quad-\frac{2 l+1}{4 l^{2}} \sum_{k=l n+1}^{m}\left(r_{i}^{\prime}(\tau) \cdot r_{k}(\tau)\right)\left(r_{j}^{\prime}(\tau) \cdot r_{k}(\tau)\right)=0, \\
& \quad(l-1) n+1 \leqq i, j \leqq l n
\end{aligned}
$$

(37) $\frac{l+1}{l} \sum_{k=l n+1}^{m}\left(r_{i}^{\prime}(\tau) \cdot r_{k}(\tau)\right) \frac{\partial \psi_{l+1}}{\partial \xi_{k}}$

$$
\begin{aligned}
= & \frac{1}{2 l} \sum_{j=l n+1}^{m}\left\{\left(r_{i}^{\prime}(\tau) \cdot r_{j}(\tau)\right)^{\prime}+(l-1) p_{l}(\tau)\left(r_{i}(\tau)^{\prime} \cdot r_{j}(\tau)\right)\right\} \xi_{j} \\
& +\frac{1}{2 l} \sum_{j, k=l n+1}^{m}\left(r_{i}^{\prime}(\tau) \cdot r_{k}(\tau)\right)\left(r_{k}^{\prime}(\tau) \cdot r_{j}(\tau)\right) \xi_{j} \\
& +\beta_{i}^{\prime}(\tau)-p_{l}(\tau) \beta_{i}(\tau)+\frac{l-2}{l-1} q_{l-1}(\tau) \beta_{i-n}(\tau), \\
& (l-1) n+1 \leqq i \leqq l n
\end{aligned}
$$


and

$$
\begin{gathered}
\text { (38) } \frac{\partial \psi_{l+1}}{\partial \tau}-\Delta_{\xi} \psi_{l+1}-\sum_{k=l n+1}^{m} \frac{\partial \psi_{l+1}}{\partial \xi_{k}}\left(\frac{\partial \psi_{l+1}}{\partial \xi_{k}}-\sum_{j=l n+1}^{m}\left(r_{k}^{\prime}(\tau) \cdot r_{j}(\tau)\right) \xi_{j}\right) \\
+\frac{2 l-1}{4 l^{2}} \sum_{i=(l-1) n+1}^{l n} \sum_{j, k=l n+1}^{m}\left(r_{i}^{\prime}(\tau) \cdot r_{j}(\tau)\right)\left(r_{i}^{\prime}(\tau) \cdot r_{k}(\tau)\right) \xi_{j} \xi_{k} \\
+\frac{l-1}{l} \sum_{i=(l-1) n+1}^{l n} \sum_{j=l n+1}^{m} \beta_{i}(\tau)\left(r_{i}^{\prime}(\tau) \cdot r_{j}(\tau)\right) \xi_{j}=0 .
\end{gathered}
$$

Let $P_{l}=P_{l}(\tau)$ be the orthogonal projection of $\mathbb{R}^{m}$ to the orthogonal complement of the subspace generated by $\left\{r_{1}(\tau), \ldots, r_{l n}(\tau)\right\}$. By (36) and (13), we have

$$
P_{l} r_{i}^{\prime} \cdot P_{l} r_{j}^{\prime}=q_{l}^{2} \delta_{i j}, \quad(l-1) n+1 \leqq i, j \leqq l n .
$$

We shall show that

$$
P_{l} r_{i}^{\prime}=r_{i}^{\prime}+q_{l-1} r_{i-n}, \quad(l-1) n+1 \leqq i \leqq l n .
$$

By recalling the definition of $P_{l},(34)$ implies

$$
P_{l} r_{i}^{\prime}=r_{i}^{\prime}-\sum_{j=1}^{(l-1) n}\left(r_{i}^{\prime} \cdot r_{j}\right) r_{j}
$$

If $1 \leqq j \leqq(l-1) n$, then by $(14)$,

$$
r_{j}^{\prime}= \begin{cases}q_{1} r_{j+n}, & 1 \leqq j \leqq n, \\ q_{k} r_{j+n}-q_{k-1} r_{j-n}, & (k-1) n+1 \leqq j \leqq k n, 2 \leqq k \leqq l-1,\end{cases}
$$

and so

$$
\begin{aligned}
r_{i}^{\prime} \cdot r_{j}=-r_{i} \cdot r_{j}^{\prime}=-q_{l-1} \delta_{i, j+n}, & (l-1) n+1 \leqq i \leqq l n, 1 \leqq j \leqq(l-1) n .
\end{aligned}
$$

Thus (40) holds.

If $q_{l}(t) \neq 0$ for all $t \in I$, then (39) and (41) imply that $r_{1}(\tau), \ldots, r_{(l+1) n}(\tau)$ defined in $(14)$ are orthonormal $C^{\infty}$-vectors of $\mathbb{R}^{m}$ on $I$ where

$$
r_{i+n}(\tau)=\frac{1}{q_{l}(\tau)}\left(r_{i}^{\prime}(\tau)+q_{l-1}(\tau) r_{i-n}(\tau)\right), \quad(l-1) n+1 \leqq i \leqq \ln .
$$


In the case of $m>(l+1) n$, we choose arbitrary $C^{\infty}$-vectors $r_{(l+1) n+1}(\tau), \ldots$, $r_{m}(\tau)$ such that $\left\{r_{1}(\tau), \ldots, r_{m}(\tau)\right\}$ forms an orthonormal basis of $\mathbb{R}^{m}$ for each $t \in I$. Then we have

$$
r_{i}^{\prime}(\tau) \cdot r_{j}(\tau)=q_{l}(\tau) \delta_{i+n, j} \quad(l-1) n+1 \leqq i \leqq l n, l n+1 \leqq j \leqq m .
$$

From (35) follows

$$
\begin{aligned}
& \psi_{l}\left(\tau, \xi_{(l-1) n+1}, \ldots, \xi_{m}\right) \\
& \qquad \begin{array}{l}
\sum_{i=(l-1) n+1}^{l n}\left(\frac{1}{4} p_{l}(\tau) \xi_{i}^{2}-\frac{1}{2 l} q_{l}(\tau) \xi_{i} \xi_{i+n}+\beta_{i}(\tau) \xi_{i}+\rho_{i}(\tau)\right) \\
+\psi_{l+l}\left(\tau, \xi_{l n+1}, \ldots, \xi_{m}\right)
\end{array}
\end{aligned}
$$

which implies

$$
\begin{aligned}
\log \varphi(\tau, \xi) & \\
=\sum_{k=1}^{l} \sum_{i=(k-1) n+1}^{k n}\left(\frac{1}{4} p_{k}(\tau) \xi_{i}^{2}\right. & \left.+\frac{1}{2 k} q_{k}(\tau) \xi_{i} \xi_{i+n}+\beta_{i}(\tau) \xi_{i}+\rho_{i}(\tau)\right) \\
& +\psi_{l+1}\left(\tau, \xi_{l n+1}, \ldots, \xi_{m}\right) .
\end{aligned}
$$

From (37) and (38) follow

$$
\begin{aligned}
\frac{\partial \psi_{l+1}}{\partial \xi_{i}}=\frac{1}{2(l+1)}\left(\frac{q_{l}^{\prime}(\tau)}{q_{l}(\tau)}-(l-1) p_{l}(\tau)\right) \xi_{i} & \\
+\frac{1}{2(l+1)} \sum_{j=(l+1) n+1}^{m}\left(r_{i}^{\prime}(\tau) \cdot r_{j}(\tau)\right) \xi_{j}+\beta_{i}(\tau), & \quad l n+1 \leqq i \leqq(l+1) n,
\end{aligned}
$$

and

$$
\begin{gathered}
\frac{\partial \psi_{l+1}}{\partial \tau}-\Delta_{\xi} \psi_{l+1}-\sum_{k=l n+1}^{m} \frac{\partial \psi_{l+1}}{\partial \xi_{k}}\left(\frac{\partial \psi_{l+1}}{\partial \xi_{k}}-\sum_{j=l n+1}^{m}\left(r_{k}^{\prime}(\tau) \cdot r_{j}(\tau)\right) \xi_{j}\right) \\
+\sum_{i=l n+1}^{(l+1) n}\left(\frac{2 l-1}{4 l^{2}} q_{l}(\tau)^{2} \xi_{i}^{2}+\frac{l-1}{l} q_{l}(\tau) \beta_{i-n}(\tau) \xi_{i}\right)=0
\end{gathered}
$$

Assume $q_{l}(t)=0$ for all $t \in I$. Then (39) gives

$$
P_{l} r_{i}^{\prime}=0, \quad(l-1) n+1 \leqq i \leqq l n .
$$


This and (40) show

$$
r_{i}^{\prime}(\tau)=-q_{l-1}(\tau) r_{i-n}(\tau), \quad(l-1) n+1 \leqq i \leqq l n .
$$

Substituting this into (35), we have

$$
\begin{aligned}
\psi_{l}\left(\tau, \xi_{(l-1) n+1}, \ldots, \xi_{m}\right)=\sum_{i=(l-1) n+1}^{l n}\left(\frac{1}{4} p_{l}(\tau) \xi_{i}^{2}+\beta_{i}(\tau) \xi_{i}+\rho_{i}(\tau)\right) & \\
& +\psi_{l+1}\left(\tau, \xi_{l n+1}, \ldots, \xi_{m}\right)
\end{aligned}
$$

which implies

$$
\begin{aligned}
& \log \varphi(\tau, \xi) \\
& =\sum_{k=1}^{l-1} \sum_{i=(k-1) n+1}^{k n}\left(\frac{1}{4} p_{k}(\tau) \xi_{i}^{2}+\frac{1}{2 k} q_{k}(\tau) \xi_{i} \xi_{i+n}+\beta_{i}(\tau) \xi_{i}+\rho_{i}(\tau)\right) \\
& \quad+\sum_{i=(l-1) n+1}^{l n}\left(\frac{1}{4} p_{l}(\tau) \xi_{i}^{2}+\beta_{i}(\tau) \xi_{i}+\rho_{i}(\tau)\right)+\psi_{l+1}\left(\tau, \xi_{l n+1}, \ldots, \xi_{m}\right) .
\end{aligned}
$$

From (38) follows

$$
\frac{\partial \psi_{l+1}}{\partial \tau}-\Delta_{\xi} \psi_{l+1}-\left|\nabla_{\xi} \psi_{l+1}\right|^{2}+\sum_{j, k=l n+1}^{m}\left(r_{k}^{\prime}(\tau) \cdot r_{j}(\tau)\right) \xi_{j} \frac{\partial \psi_{l-1}}{\partial \xi_{k}}=0 .
$$

Thus the assertion for $l$ is shown.

Proof of Theorem 7. For each $t \in D$, there exists a positive integer $l \leqq m / n$ such that $q_{l}(t)=0$. In fact, if $q_{1}(t) \neq 0, \ldots, q_{k}(t) \neq 0$, then by Lemma $9,(k+1) n \leqq m$.

Assume that $q_{1} \neq 0, \ldots, q_{l-1} \neq 0$ and $q_{l}=0$ on an open interval $I$. Then by (14) and (16), we obtain $n$ systems of linear differential equations:

$$
\begin{aligned}
(42) \frac{d}{d t}\left(\begin{array}{c}
r_{i} \\
r_{n+i} \\
\vdots \\
r_{(l-1) n+i}
\end{array}\right) & =\left(\begin{array}{cccc}
0 & q_{1} & & 0 \\
-q_{1} & 0 & \ddots & \\
& \ddots & \ddots & q_{l-1} \\
0 & & -q_{l-1} & 0
\end{array}\right)\left(\begin{array}{c}
r_{i} \\
r_{n+i} \\
\vdots \\
r_{(l-1) n+i}
\end{array}\right) \\
& =: Q\left(\begin{array}{c}
r_{i} \\
r_{n+i} \\
\vdots \\
r_{(l-1) n+i}
\end{array}\right)
\end{aligned}
$$


for $1 \leqq i \leqq n$. Fix arbitrary $t_{0} \in I$ and let $S(t)=\left(s_{j k}(t)\right)_{j, k=1}^{l}$ be the solution of the initial value problem

$$
\left\{\begin{array}{l}
\frac{d}{d t} S(t)=Q(t) S(t), \\
S\left(t_{0}\right)=I_{l},
\end{array}\right.
$$

where $I_{l}$ is the $(l, l)$ unit matrix. Then $S(t)$ is an orthogonal matrix for every $t \in I$, because $Q(t)$ is skew symmetric. Then by (42), we have

$$
\left(\begin{array}{c}
r_{i}(t) \\
r_{n+i}(t) \\
\vdots \\
r_{(l-1) n+i}(t)
\end{array}\right)=S(t)\left(\begin{array}{c}
r_{i}\left(t_{0}\right) \\
r_{n+i}\left(t_{0}\right) \\
\vdots \\
r_{(l-1) n+i}\left(t_{0}\right)
\end{array}\right), \quad 1 \leqq i \leqq n .
$$

This means that $r_{1}(t), r_{2}(t), \ldots, r_{l n}(t)$ are contained in the $l n$-dimensional space $V$ spanned by the constant vectors $r_{1}\left(t_{0}\right), r_{2}\left(t_{0}\right), \ldots, r_{l n}\left(t_{0}\right)$ for every $t$. Therefore we can choose constant vectors $r_{l n+1}, \ldots, r_{m}$ which are the orthonormal basis of the orthogonal complement of $V$. Put $x_{j}=r_{j}\left(t_{0}\right) \cdot x$, $1 \leqq j \leqq m$ for $x \in \mathbb{R}^{m}$. Then

$$
\xi_{(j-1) n+i}=\sum_{k=1}^{l} s_{j k}(t) x_{(k-1) n+i}, \quad 1 \leqq i \leqq n, \quad 1 \leqq j \leqq l
$$

and if $m \geqq \ln +1$,

$$
\xi_{j}=x_{j}, \quad l n+1 \leqq j \leqq m .
$$

Then $\psi_{l+1}$ is a $C^{\infty}$-function of $t, x_{l n+1}, \ldots, x_{m}$ and so the equation (18) reduces to

$$
\frac{\partial \psi_{l+1}}{\partial t}-\Delta \psi_{l+1}-\left|\nabla \psi_{l+1}\right|^{2}=0
$$

Therefore $\varphi_{l+1}\left(t, x_{l n+1}, \ldots, x_{m}\right)=\exp \psi_{l+1}$ is a positive caloric function (in the case of $m=l n, \psi_{l+1}$ is equal to a constant). From (20) follows

$$
f_{i}=\sum_{k=1}^{l} \lambda(t) s_{1 k}(t) x_{(k-1) n+i}+b_{i}(t)
$$

where $\lambda(t)=\sqrt{f_{0}^{\prime}(t)}$. On the other hand, by (17) and (44) we have 
$\log \varphi$

$$
\begin{aligned}
& =\sum_{i=1}^{n}\left[\sum_{j, k=1}^{l} \frac{1}{4} u_{j k}(t) x_{(j-1) n+i} x_{(k-1) n+i}+\sum_{j=1}^{l} \frac{1}{2} v_{i j}(t) x_{(j-1) n+i}+w_{i}(t)\right] \\
& \quad+\psi_{l+1},
\end{aligned}
$$

where

$$
u_{i j}=\sum_{k=1}^{l} p_{k} s_{k i} s_{k j}+\sum_{k=1}^{l-1} \frac{q_{k}}{k}\left(s_{k i} s_{k+1, j}+s_{k+1, i} s_{k j}\right), \quad 1 \leqq i, j \leqq l,
$$

and

$$
v_{i j}=\sum_{k=1}^{l} 2 \beta_{(k-1) n+i} s_{k j}, \quad w_{i}=\sum_{k=1}^{l} \rho_{(k-1) n+i}, \quad 1 \leqq i \leqq n, 1 \leqq j \leqq l .
$$

Put

$$
\begin{aligned}
\text { (45) } g_{i 1}\left(t, x_{1}, \ldots, x_{l}\right) & =\sum_{j=1}^{l} \lambda(t) s_{1 j}(t) x_{j}+b_{i}(t), \quad 1 \leqq i \leqq n \\
g_{i}\left(t, x_{1}, \ldots, x_{l}\right) & =\left(f_{0}(t), g_{i 1}\left(t, x_{1}, \ldots, x_{l}\right)\right), \quad 1 \leqq i \leqq n \\
\varphi_{i}\left(t, x_{1}, \ldots, x_{l}\right) & =\exp \left[\sum_{j, k=1}^{l} \frac{1}{4} u_{j k}(t) x_{j} x_{k}+\sum_{j=1}^{l} \frac{1}{2} v_{i j}(t) x_{j}+w_{i}(t)\right], \\
1 \leqq i \leqq n . & 1 \leqq i \leqq
\end{aligned}
$$

Then

$$
\begin{aligned}
& f_{i}(t, x)=g_{i 1}\left(t, x_{i}, x_{n+i}, \ldots, x_{(l-1) n+i}\right), \\
& \varphi(t, x)=\varphi_{l+1} \prod_{i=1}^{n} \varphi_{i}\left(t, x_{i}, x_{n+i}, \ldots, x_{(l-1) n+i}\right) .
\end{aligned}
$$

We shall prove that each pair $\left(g_{i}, \varphi_{i}\right), 1 \leqq i \leqq n$ is a caloric morphism from $I \times \mathbb{R}^{l}$ to $\mathbb{R}^{1+1}$. By $H g_{i 1}=\partial g_{i 1} / \partial t$ and (43), we have

$$
\begin{aligned}
H g_{i 1} & =\sum_{j=1}^{n}\left(\lambda^{\prime}(t) s_{1 j}(t) x_{j}+\lambda(t) s_{1 j}^{\prime}(t) x_{j}\right)+b_{i}^{\prime}(t) \\
& =\sum_{j=1}^{n}\left(\lambda^{\prime}(t) s_{1 j}(t) x_{j}+\lambda(t) q_{1}(t) s_{2 j}(t) x_{j}\right)+b_{i}^{\prime}(t) .
\end{aligned}
$$


On the other hand,

$$
\begin{aligned}
2 \nabla \log \varphi_{i} \cdot \nabla g_{i 1} & =\sum_{j, k=1}^{l} \frac{1}{2} \lambda\left(u_{j k} s_{1 k}+u_{k j} s_{1 k}\right) x_{j}+\sum_{j=1}^{l} \lambda v_{i j} s_{1 j} \\
& =\sum_{j=1}^{l} \lambda\left(p_{1} s_{1 j} x_{j}+q_{1} s_{2 j} x_{j}+2 \beta_{i}\right),
\end{aligned}
$$

because $u_{i j}=u_{j i}$ and $S$ is orthogonal. Hence

$$
H g_{i 1}=2 \nabla \log \varphi_{i} \cdot \nabla g_{i 1}, \quad 1 \leqq i \leqq n
$$

Since $f_{0}^{\prime}=\lambda^{2}$,

$$
\frac{d f_{0}}{d t}=\left|\nabla g_{i 1}\right|^{2}
$$

By the assumption, $\varphi(t, x)$ and $\varphi_{l+1}$ are caloric functions, $\varphi_{l+1}$ is independent of $x_{1}, \ldots, x_{l n}$ and

$$
\prod_{i=1}^{n} \varphi_{i}\left(t, x_{i}, x_{n+1}, \ldots, x_{(l-1) n+i}\right)
$$

is a caloric function. Hence we have

$$
\sum_{i=1}^{n}\left(K \varphi_{i}\right)\left(t, x_{i}, x_{n+i}, \ldots, x_{(l-1) n+i}\right)=0
$$

where $K \varphi_{i}=\left(1 / \varphi_{i}\right) H \varphi_{i}$. We have also $K \varphi_{i}=\left(\partial \log \varphi_{i} / \partial t\right)-\Delta \log \varphi_{i}-$ $\left|\nabla \log \varphi_{i}\right|^{2}$. Comparing the coefficients with respect to $x_{j}$, we see that $K \varphi_{i}$ depends only on $t$. Therefore

$$
\frac{\partial \log \varphi_{i}}{\partial t}-\Delta \log \varphi_{i}-\left|\nabla \log \varphi_{i}\right|^{2}=\sum_{j=1}^{l}\left(\rho_{(j-1) n+i}^{\prime}-\frac{1}{2} u_{j j}-\frac{1}{4} v_{i j}^{2}\right)
$$

Since

$$
\left(\begin{array}{ccc}
u_{11} & \ldots & u_{1 l} \\
\vdots & \ddots & \vdots \\
u_{l 1} & \ldots & u_{l l}
\end{array}\right)={ }^{t} S\left(\begin{array}{cccc}
p_{1} & q_{1} & & 0 \\
q_{1} & p_{2} & \ddots & \\
& \ddots & \ddots & \frac{q_{l-1}}{l-1} \\
0 & & \frac{q_{l-1}}{l-1} & p_{l}
\end{array}\right) S
$$


and

$$
\left(v_{i 1}, \ldots, v_{i l}\right)=2\left(\beta_{i}, \beta_{n+i}, \ldots, \beta_{(l-1) n+i}\right) S
$$

we have

$$
\sum_{j=1}^{l}\left(\rho_{(j-1) n+i}^{\prime}-\frac{1}{2} u_{j j}-\frac{1}{4} v_{i j}^{2}\right)=\sum_{j=1}^{l}\left(\rho_{(j-1) n+i}^{\prime}-\frac{p_{j}}{2}-\beta_{(j-1) n+i}^{2}\right)=0
$$

by the definition of $\rho_{j}$ in (15). Therefore each $\varphi_{i}$ is a positive caloric function. Thus $\left(g_{i}, \varphi_{i}\right)$ is a caloric morphism. By $(45)$ and $(46)$, each $\left(g_{i}, \varphi_{i}\right)$ satisfies the assumption of Lemma 8 . Therefore there exist a positive integer $k \leqq l$, an orthogonal coordinate of $\mathbb{R}^{m}$ denoted by $\left(x_{1}, \ldots, x_{m}\right)$ again and positive caloric functions $h_{i}=h_{i}\left(t, x_{k n+i}, \ldots, x_{(l-1) n+i}\right), 1 \leqq i \leqq n$ (in the case of $k=l, h_{1}, \ldots, h_{n}$ are positive constants) such that $f$ and $\varphi$ are of form (1) or (2) with four families $\alpha_{i}, 1 \leqq i \leqq k, \beta_{i}, 1 \leqq i \leqq k, \delta_{i}, 0 \leqq i \leqq n$ and $\gamma_{i j}$, $1 \leqq i \leqq n, 1 \leqq j \leqq k$ of real numbers satisfying $\alpha_{i}>0$ and $\beta_{i} \neq \beta_{j}, i \neq j$ :

$$
\begin{aligned}
f_{0}(t) & =\sum_{j=1}^{k} \frac{\alpha_{j}^{2}}{\beta_{j}-t}+\delta_{0}, \\
f_{i}(t, x) & =g_{i 1}\left(t, x_{i}, \ldots, x_{(l-1) n+i}\right)=\sum_{j=1}^{k} \frac{\alpha_{j}}{\beta_{j}-t}\left(x_{(j-1) n+i}+\gamma_{i j}\right)+\delta_{i} \\
\varphi(t, x) & =\varphi_{l+1} \prod_{i=1}^{n} \varphi_{i}\left(t, x_{i}, \ldots, x_{(l-1) n+i}\right) \\
& =\varphi_{l+1} \prod_{i=1}^{n} h_{i} \prod_{j=1}^{k} \frac{1}{\left|\beta_{j}-t\right|^{1 / 2}} \exp \frac{\left(x_{(j-1) n+i}+\gamma_{i j}\right)^{2}}{4\left(\beta_{j}-t\right)}
\end{aligned}
$$

$$
\begin{aligned}
f_{0}(t)= & \alpha_{1}^{2} t+\sum_{1<j \leqq k} \frac{\alpha_{j}^{2}}{\beta_{j}-t}+\delta_{0} \\
f_{i}(t, x) & =g_{i 1}\left(t, x_{i}, \ldots, x_{(l-1) n+i}\right) \\
& =\alpha_{1}\left(x_{i}+\gamma_{i 1} t\right)+\sum_{1<j \leqq k} \frac{\alpha_{j}}{\beta_{j}-t}\left(x_{(j-1) n+i}+\gamma_{i j}\right)+\delta_{i},
\end{aligned}
$$




$$
\begin{aligned}
\varphi(t, x)= & \varphi_{l+1} \prod_{i=1}^{n} \varphi_{i}\left(t, x_{i}, \ldots, x_{(l-1) n+i}\right) \\
= & \varphi_{l+1} \prod_{i=1}^{n} h_{i} \exp \left[\frac{\gamma_{i 1}^{2}}{4} t+\frac{\gamma_{i 1}}{2} x_{i}\right] \\
& \times \prod_{1<j \leqq k} \frac{1}{\left|\beta_{j}-t\right|^{1 / 2}} \exp \frac{\left(x_{(j-1) n+i}+\gamma_{i j}\right)^{2}}{4\left(\beta_{j}-t\right)} .
\end{aligned}
$$

Put $h=\varphi_{l+1} h_{1} \cdots h_{n}$. Then $h=h\left(t, x_{k n+1}, \ldots, x_{m}\right)$ is a positive caloric function. We obtain the required form of $(f, \varphi)$ on $D \cap\left(I \times \mathbb{R}^{m}\right)$. Since $f_{0}$ is of $C^{\infty}$, the form of $(f, \varphi)$ holds on the closure $\bar{I}$ of $I$, if $\bar{I}$ is contained in the interval where $f_{0}$ is defined. Thus $(f, \varphi)$ has the required form on each open interval where $q_{1}>0, \ldots, q_{l-1}>0$. Fix an open interval $I$ such that $q_{1}>0, \ldots, q_{l-2}>0$. The analyticity of $f_{0}$ and (13) implies that $q_{l-1}$ is an analytic function on $I$. Therefore, the zero-points of $q_{l-1}$ is discrete, which is denoted by $\left\{\sigma_{\nu}\right\}_{\nu=M}^{N}(M, N$ may be $-\infty, \infty$, respectively). For each $\nu$, $f_{0}$ is of form

$$
f_{0}(t)=\left\{\begin{array}{l}
\sum_{j=1}^{k} \frac{\alpha_{j}^{2}}{\beta_{j}-t}+\delta_{0}, \quad t \in\left(\sigma_{\nu-1}, \sigma_{\nu}\right], \\
\sum_{j=1}^{\tilde{k}} \frac{\tilde{\alpha}_{j}^{2}}{\tilde{\beta}_{j}-t}+\tilde{\delta}_{0}, \quad t \in\left[\sigma_{\nu}, \sigma_{\nu+1}\right),
\end{array}\right.
$$

in the case of (1). Then $\tilde{k}=k, \tilde{\alpha}_{j}=\alpha_{j}, \tilde{\beta}_{j}=\beta_{j}$ and $\tilde{\delta}_{0}=\delta_{0}$, because $f_{0}$ is of $C^{\infty}$. Therefore $(f, \varphi)$ has the required form on each interval where $q_{1}>$ $0, \ldots, q_{l-2}>0$. In the case of $(2)$, the same argument holds. Consequently, $(f, \varphi)$ is of a required form on $D$. This completes the proof of Theorem 7 .

Corollary 10. Let $(f, \varphi)$ be the same as in Theorem 7 . Then $(f, \varphi)$ is equal to the composition of a the direct sum of $k$ caloric morphisms of $\mathbb{R}^{n+1}$ and a projection $\mathbb{R}^{m+1} \rightarrow \mathbb{R}^{k n+1}$.

Proof. In the case of (I), we put

$$
g_{j 0}(t)= \begin{cases}\frac{\alpha_{1}^{2}}{\beta_{1}-t}+\delta_{0}, & j=1, \\ \frac{\alpha_{j}^{2}}{\beta_{j}-t}, & j>1\end{cases}
$$




$$
\begin{aligned}
& g_{j i}\left(t, x_{1}, \ldots, x_{n}\right)= \begin{cases}\frac{\alpha_{1}}{\beta_{1}-t}\left(x_{i}+\gamma_{i j}\right)+\delta_{i}, & j=1, \\
\frac{\alpha_{j}}{\beta_{j}-t}\left(x_{i}+\gamma_{i j}\right), & j>1,\end{cases} \\
& \varphi_{j}\left(t, x_{1}, \ldots, x_{n}\right)=\frac{1}{\left|\beta_{j}-t\right|^{n / 2}} \exp \sum_{i=1}^{n} \frac{\left(x_{i}+\gamma_{i j}\right)^{2}}{4\left(\beta_{j}-t\right)},
\end{aligned}
$$

for $1 \leqq i \leqq n$ and $1 \leqq j \leqq k$. In the case of (II), we put

$$
\begin{aligned}
g_{j 0}(t)=\left\{\begin{array}{l}
\alpha_{1}^{2} t+\delta_{0}, \quad j=1, \\
\frac{\alpha_{j}^{2}}{\beta_{j}-t}, \quad j>1,
\end{array}\right. \\
g_{j i}\left(t, x_{1}, \ldots, x_{n}\right)= \begin{cases}\alpha_{1}\left(x_{i}+\gamma_{i 1} t\right)+\delta_{1}, \quad j=1, \\
\frac{\alpha_{j}}{\beta_{j}-t}\left(x_{i}+\gamma_{i j}\right), \quad j>1, \\
\exp \sum_{i=1}^{n}\left[\frac{\gamma_{i 1}^{2}}{4} t+\frac{\gamma_{i 1}}{2} x_{i}\right], & j=1, \\
\frac{1}{\left|\beta_{j}-t\right|^{n / 2}} \exp \sum_{i=1}^{n} \frac{\left(x_{i}+\gamma_{i j}\right)^{2}}{4\left(\beta_{j}-t\right)}, & j>1,\end{cases}
\end{aligned}
$$

for $1 \leqq i \leqq n$ and $1 \leqq j \leqq k$. Then each pair $\left(g_{j}, \varphi_{j}\right)=\left(\left(g_{j 0}, \ldots, g_{j n}\right), \varphi_{j}\right)$, $1 \leqq j \leqq k$ is a caloric morphism. $\left(g_{1}, \varphi_{1}\right)$ is defined on $\mathbb{R}^{n} \backslash\left\{t \neq \beta_{1}\right\}$ in the case of $(\mathrm{I})$ and on $\mathbb{R}^{n}$ in the case of $(\mathrm{I})$. For $j>1,\left(g_{j}, \varphi_{j}\right)$ is defined on $\mathbb{R}^{n} \backslash\left\{t \neq \beta_{j}\right\}$. Let $(p, \psi)$ be the projection $\mathbb{R}^{m+1} \rightarrow \mathbb{R}^{k n+1}$ such that $p_{0}(t)=t, p_{i}\left(t, x_{1}, \ldots, x_{m}\right)=x_{i}, 1 \leqq i \leqq k n$ and $\psi\left(t, x_{1}, \ldots, x_{m}\right)=$ $h\left(t, x_{k n+1}, \ldots, x_{m}\right)$. Then $(f, \varphi)$ is equal to the composition of the direct sum of $\left(g_{1}, \varphi_{1}\right), \ldots,\left(g_{k}, \varphi_{k}\right)$ and $(p, \psi)$.

\section{REFERENCES}

[1] P. Appell, Sur l'équation $\partial^{2} z / \partial x^{2}-\partial z / \partial y$ et la théorie de la chaleur, J. Math. Pures Appl., 8 (1892), 186-216.

[2] B. Fuglede, Harmonic Morphisms Between Riemannian Manifolds, Ann. Inst. Fourier(Grenoble), 28 (1978), no. 2, 107-144.

[3] B. Fuglede, Harmonic Morphisms, Complex Analysis, Joensuu 1978, Lecture Notes in Math., 747 (1979), Springer-Verlag, Berlin (1979), 123-131.

[4] B. Fuglede, Harmonic morphisms between semi-riemannian manifolds, Ann. Acad. Sci. Fenn. Math., 21 (1996), 31-50. 
[5] F. W. Gehring and H. Haahti, The transformations which preserve the harmonic functions, Ann. Acad. Sci. Fenn. Ser. A I Math., 293 (1960), 1-12.

[6] T. Ishihara, A mapping of Riemannian manifolds which preserves harmonic functions, J. Math. Kyoto Univ., 19 (1979), no. 2, 215-229.

[7] H. Leutwiler, On the Appell Transformation, Potential Theory (1988), Plenum, New York, 215-222.

[8] K. Shimomura, Caloric morphisms and a generalization of the Appell transformation, Proceedings of the Seventh International Colloquium on Differential Equations (1997), VSP Press, Netherlands, 389-394.

[9] F. Trèves, Topological Vector Spaces, Distributions and Kernels, Academic Press, New York, 1967.

Department of Mathematical Sciences

Ibaraki University

Mito, Ibaraki, 310

Japan

shimomur@mito.ipc.ibaraki.ac.jp 